\title{
Structure of the virtual Compton amplitude with an additional final-state meson in the extended Bjorken region
}

\author{
Johannes Blümlein* \\ Deutsches Elektronen-Synchrotron, DESY-Zeuthen, Platanenallee 6, D-15735 Zeuthen, Germany \\ Jörg Eilers ${ }^{\dagger}$ and Bodo Geyer ${ }^{\star}$ \\ Center for Theoretical Studies and Institute of Theoretical Physics, Leipzig University, Augustusplatz 10, D-04109 Leipzig, Germany \\ Dieter Robaschik ${ }^{\S}$ \\ BTU Cottbus, Fakultät 1, Postfach 101344, D-03013 Cottbus, Germany
}

(Received 10 August 2001; published 15 February 2002)

\begin{abstract}
Using the framework of the nonlocal light-cone expansion a systematic study is performed for the structure of the twist- 2 contributions to the virtual Compton amplitude in polarized deep-inelastic nonforward scattering for general nucleon spin with an additional scalar meson in the final state. A useful kinematic parametrization allowing for appropriate triple-valued off-forward parton distribution amplitudes is given. One-variable amplitudes being adapted to the fixed parameters of the extended Bjorken region are introduced by decomposing the Compton amplitude into collinear and noncollinear components. These amplitudes obey Wandzura-Wilczekand Callan-Gross-like relations. The evolution equations for all the distribution amplitudes are determined showing that the additional meson momentum does not appear in the evolution kernels. The generalization to $n$ outgoing mesons is given.
\end{abstract}

DOI: 10.1103/PhysRevD.65.054029

PACS number(s): 13.85.Hd, 13.88.+e

\section{INTRODUCTION}

Compton scattering of a virtual photon off a hadron,

$$
\gamma^{*}\left(q_{1}\right)+H\left(p_{1}\right) \rightarrow \gamma^{*}\left(q_{2}\right)+H\left(p_{2}\right)
$$

is an important process in quantum chromodynamics. This general process covers a series of different reactions through which a variety of inclusive information on the shortdistance structure of nucleons becomes accessible at large spacelike virtualities. It is also closely connected to the spin problem of the nucleon. The case of forward scattering $p_{1}$ $=p_{2}=p$ describes deep inelastic scattering (DIS) off unpolarized or polarized targets which is widely discussed in the literature, see, e.g., $[1,2]$, and $p_{1} \neq p_{2}$ corresponds to the generic (ordinary) nonforward virtual Compton scattering [3-7], for recent reviews see [8]. In the special case $q_{2}^{2}=0$, i.e., when the outgoing photon is real, this process is called deeply virtual Compton scattering (DVCS).

Experimental results on polarized and unpolarized (deeply virtual) Compton scattering were reported in [9-13]. The kinematic domain of some of these investigations is bound to rather low values of $Q^{2}$. Experimentally the final state in deep-inelastic nonforward scattering contains, aside from the (virtual) photon and final-state hadron, Eq. (1.1), a series of other hadrons, which even may emerge at the amplitude level. The latter process is much more likely and of greater

\footnotetext{
*Electronic address: johannes.bluemlein@ desy.de

${ }^{\dagger}$ Electronic address: eilers@itp.uni-leipzig.de

*Electronic address: geyer@itp.uni-leipzig.de

${ }^{\S}$ Electronic address: drobasch@physik.tu-cottbus.de
}

practical importance than that of a single isolated hadron $\gamma_{1}^{*}+H_{1} \rightarrow \gamma_{2}^{*}+H_{2}$, which was studied before [3-7].

In this paper we extend the description given in the ordinary nonforward case in Refs. [5-7] to physical processes with an outgoing scalar meson,

$$
\gamma^{*}\left(q_{1}\right)+H\left(p_{1}\right) \rightarrow \gamma^{*}\left(q_{2}\right)+H\left(p_{2}\right)+M(k),
$$

to investigate which of the properties derived in Refs. [5-7] remain valid in the more general situation and which are changing to account for more realistic experimental situations which allow for additional studies of distribution functions emerging in nonforward scattering [14].

If one looks for a diagrammatic representation of the amplitude for the process (1.2) then even in the special kinematics of the Bjorken region for $\left(p_{2}+k-p_{1}\right)^{2}$ being small, there appear different production mechanisms. However, if one has in mind $p_{2}^{2}=M^{2}, k^{2}=m^{2}$, and $p_{2} \cdot k \ll M^{2}$ then soft processes between the two final particles and the incoming particle $\left|p_{1}\right\rangle$ are essential and we have to use a generalized distribution amplitude $\left\langle p_{2}, k|O| p_{1}\right\rangle$. For other cases one may try to make models which use wave functions of the nucleon $\left\langle p_{2}\right|$ and the meson $\langle k|$.

The Compton amplitude for the process (1.2) is given by

$$
\begin{aligned}
T_{\mu \nu}\left(P_{+}, P_{-}, k ; q\right)= & i \int d^{4} x e^{i q x}\left\langle p_{2}, S_{2} ; k, 0\right| \\
& \times R T\left[J_{\mu}\left(\frac{x}{2}\right) J_{\nu}\left(-\frac{x}{2}\right) \mathcal{S}\right]\left|p_{1}, S_{1}\right\rangle
\end{aligned}
$$

where 


$$
P_{ \pm} \equiv P_{f} \pm P_{i}=p_{2} \pm p_{1}+k, \quad q=\frac{1}{2}\left(q_{1}+q_{2}\right),
$$

and $k$ are chosen as independent kinematic variables. As usual $p_{1}\left(p_{2}\right)$ and $q_{1}\left(q_{2}\right)$ denote the four-momenta of the incoming (outgoing) nucleons and photons, respectively. $S_{1}$ and $S_{2}$ are the spins of these nucleons and $k$ is the momentum of the outgoing scalar meson. $p_{ \pm}=p_{2} \pm p_{1}$ denote the independent kinematic variables in the ordinary nonforward case. In principle, this set of kinematic variables can be extended to the case of $n$ outgoing (scalar) mesons of momenta $k_{i}, i=1, \ldots, n$ with $P_{f}=p_{2}+\sum_{i} k_{i}$.

In ordinary nonforward Compton scattering the generalized Bjorken region is defined by the conditions

$$
\nu \equiv q p_{+} \rightarrow \infty \quad \text { and } \quad Q^{2} \equiv-q^{2} \rightarrow \infty,
$$

keeping the variables

$$
\xi=-\frac{q^{2}}{q p_{+}} \quad \text { and } \quad \eta=\frac{q p_{-}}{q p_{+}}=\frac{q_{1}^{2}-q_{2}^{2}}{2 \nu}
$$

fixed. This definition has to be extended now by taking into account the additional momentum $k$. We define the extended Bjorken region for light-cone-dominated QCD processes by the conditions (1.5) keeping the following three variables:

$$
\xi=-\frac{q^{2}}{q P_{+}}, \quad \eta=\frac{q P_{-}}{q P_{+}}, \quad \text { and } \quad \chi=\frac{q k}{q P_{+}}
$$

fixed. Furthermore, this can be extended to the case of $n$ mesons by the obvious generalization of $P_{+}$and keeping $\chi_{i}=\left(q k_{i}\right) /\left(q P_{+}\right)$fixed. In the limit $k_{i} \rightarrow 0$, these definitions reduce to the usual generalized Bjorken region.

For completeness, we list the different kinematic domains for forward and general nonforward processes and the related scaling variables. These domains are distinguished as follows:

Bjorken region for forward scattering; fixed quantity: $\xi$ (or $x_{B}=-q_{1}^{2} / 2 q_{1} \cdot p_{1}$ ).

Generalized Bjorken region for ordinary nonforward scattering, including DVCS kinematics for $q_{2}^{2}=0$; fixed quantities: $\xi$ and $\eta$. In the special case of DVCS $\xi=-\eta$ holds.

Extended Bjorken region for nonforward scattering with a single outgoing scalar meson; fixed quantities: $\xi, \eta$, and $\chi$.

$n$-extended Bjorken region for nonforward scattering with $n$ outgoing scalar mesons; fixed quantities: $\xi, \eta$, and $\chi_{1}, \ldots, \chi_{n}$.

By conservation of momentum,

$$
p_{1}+q_{1}=p_{2}+q_{2}+\sum_{i} k_{i}
$$

one can show that $\xi$ and $\eta$ possess the same interpretation as in the ordinary nonforward case. Especially, also the relation $\eta=\left(q_{1}^{2}-q_{2}^{2}\right) / 2 \nu$ holds for processes including the outgoing mesons. The variables $\chi_{i}$ describe the momentum fractions of the mesons $k_{i}$ in the infinite momentum frame defined by the momentum $P_{+}$. In the following we restrict the consid- eration to the case of one additional final-state meson and return to the case of $n$-mesons in Sec. VII.

It is important to remark that all physical processes mentioned above are distinguished only by taking different matrix elements of the same renormalized operator [15], namely the renormalized $(R)$ time-ordered $(T)$ product

$$
\hat{T}_{\mu \nu}(x) \equiv R T\left[J_{\mu}\left(\frac{x}{2}\right) \quad J_{\nu}\left(-\frac{x}{2}\right) \mathcal{S}\right],
$$

where $J_{\mu}(x)=: \bar{\psi}(x) \gamma_{\mu} \psi(x)$ : denotes the hadronic current and $\mathcal{S}$ is the renormalized $S$ matrix. Near the lightcone, $x^{2}$ $\rightarrow 0$, this operator will be decomposed via the nonlocal operator product expansion [16] into a series of nonlocal lightray operators and the corresponding coefficient functions:

$$
\begin{aligned}
\hat{T}_{\mu \nu}(x)= & \int_{-1}^{1} d^{2} \kappa C_{\Gamma \mu \nu}\left(x^{2}, \kappa_{i} \tilde{x} ; \mu^{2}\right) R T\left[O^{\Gamma}\left(\kappa_{1} \tilde{x}, \kappa_{2} \tilde{x}\right) \mathcal{S}\right] \\
& + \text { higher order terms. }
\end{aligned}
$$

The coefficient functions are singular on the light cone. They are entire analytic functions with respect to $\kappa_{i} \tilde{x}$ resulting in a restricted integration range $-1 \leqslant \kappa_{i} \leqslant 1$. The unrenormalized light-ray operators in this expansion are given by

$$
O^{\Gamma}\left(\kappa_{1} \tilde{x}, \kappa_{2} \tilde{x}\right)=: \bar{\psi}\left(\kappa_{1} \tilde{x}\right) \Gamma U\left(\kappa_{1} \tilde{x}, \kappa_{2} \tilde{x}\right) \psi\left(\kappa_{2} \tilde{x}\right):
$$

with some specified $\Gamma$ structure of Dirac matrices and the usual path-ordered phase factor,

$$
U\left(\kappa_{1} \tilde{x}, \kappa_{2} \tilde{x}\right)=\mathrm{P} \exp \left(-i g \int_{\kappa_{2}}^{\kappa_{1}} d \tau \bar{x}^{\mu} A_{\mu}(\tau \tilde{x})\right),
$$

ensuring gauge invariance. Here $A_{\mu}$ is the gluon field, $g$ the strong coupling constant, and $\tilde{x}$ is a lightlike vector depending on $x$ via a non-null subsidiary four-vector $\zeta$,

$$
\tilde{x}=x-\frac{\zeta}{\zeta^{2}}\left((x \zeta)-\sqrt{(x \zeta)^{2}-x^{2} \zeta^{2}}\right) \quad \text { with } \quad \zeta^{2}>0
$$

In Eq. (1.10) the flavor structure has been suppressed. Eventually, in the singlet case, also operators containing the gluon field strength $F_{\mu \nu}$ and their dual $\widetilde{F}_{\mu \nu}$ have to be taken into account. The contributions which contain four or more (anti)quark fields will be denoted by "higher order terms," possibly also together with (powers of) the gluon field strength, etc. By construction, the nonlocal light-cone expansion, depending on the order of terms being taken into account, leads to a (sub)asymptotically relevant part and a well-defined remainder being less singular, see Ref. [2].

Taking matrix elements of the operators $O^{\Gamma}$ and performing a Fourier transformation in the expression (1.3) leads to the physically interesting nonperturbative distribution amplitudes. Because the operational input (1.9) for these amplitudes is the same for the different processes described above, the evolution equations of these amplitudes are determined 
by the renormalization group equations of the operators $O^{\Gamma}$ and, therefore, also by the same anomalous dimensions.

This paper is organized as follows. In Sec. II we discuss the quark-antiquark operator (2.13) as operational input for the (extended) Compton amplitude. This allows one to use the known anomalous dimensions of this operator to write down the evolution equations for the relevant distribution amplitudes used in the process including an outgoing meson. The evolution kernel required for these evolution equations is determined by the anomalous dimensions of the quarkantiquark operator and further computations of Feynman diagrams are not necessary, at least at one-loop order. Furthermore, this operator possesses a known twist decomposition given in Refs. $[17,18]$. In this paper we only determine the twist-2 part of the Compton amplitude in the simply extended Bjorken region which generalizes the twist-2 representation known for forward and ordinary nonforward scattering.

In Sec. III the twist decomposition of the matrix elements and the necessary decomposition into suitable kinematic factors is performed, thereby using the hadron equations of motion. This section also includes the definition of the distribution amplitudes used in the process considered.

In Sec. IV the twist-2 part of the Compton amplitude is calculated. It will be shown that, in the extended kinematic region, the amplitude depends on the three scaling variables $\xi, \eta$ and $\chi$ and is given by triple-valued distributions.

In Sec. V we extract integral relations contained in the Compton amplitude and Sec. VI includes the evolution equations obeyed by the distribution amplitudes.

In Sec. VII we add some remarks on generic properties of the results obtained in the preceding sections and consider the case of $n$ outgoing mesons. Section VIII contains the conclusions.
Appendix A contains the projections of the twist-2 operators on the light cone. In Appendix B we construct the helicity basis for both photons and calculate all the helicity amplitudes. These projections show in explicit form that the process (1.2) is current conserving on the level of the $S$ matrix.

\section{OPERATOR STRUCTURE}

In this section we discuss the operator structure of the Compton amplitude which will be used in the following sections. For brevity we discuss only the flavor nonsinglet case and drop all flavor structures in the operators. The construction for the flavor singlet case is to be carried out similarly.

As was mentioned above, the operator input for the Compton amplitude $T_{\mu \nu}\left(P_{ \pm}, k ; q\right)$ is given by the renormalized time-ordered product of two electromagnetic currents, Eq. (1.9). Obviously, in lowest order in the coupling constant the $S$ matrix can be set equal to one. Then, by applying the Wick theorem to this time-ordered product and approximating the quark propagator near the light cone by

$$
\begin{aligned}
S(x, m) \equiv & (i b+m) \Delta(x, m) \\
\approx & -\frac{k}{2 \pi^{2}\left(x^{2}-i \epsilon\right)^{2}}-\frac{m}{8 \pi^{2}\left(x^{2}-i \epsilon\right)}(2 i+m k) \\
& +\frac{m^{3}}{128 \pi^{2}} \ln \left(-x^{2}+i \epsilon\right)(8 i+m k),
\end{aligned}
$$

one obtains the following description of the operator $\hat{T}_{\mu \nu}(x)$ :

$$
\begin{aligned}
T\left[J_{\mu}\left(\frac{x}{2}\right) J_{\nu}\left(-\frac{x}{2}\right)\right] \approx & -x^{\alpha}\left[\frac{1}{2 \pi^{2}\left(x^{2}-i \epsilon\right)^{2}}+\frac{m^{2}}{8 \pi^{2}\left(x^{2}-i \epsilon\right)}-\frac{m^{4}}{128 \pi^{2}} \ln \left(-x^{2}+i \epsilon\right)\right] \\
& \times\left[: \bar{\psi}(x / 2) \gamma_{\mu} \gamma_{\alpha} \gamma_{\nu} \psi(-x / 2):-: \bar{\psi}(-x / 2) \gamma_{\nu} \gamma_{\alpha} \gamma_{\mu} \psi(x / 2):\right]-i m\left[\frac{1}{4 \pi^{2}\left(x^{2}-i \epsilon\right)}-\frac{m^{2}}{16 \pi^{2}} \ln \left(-x^{2}+i \epsilon\right)\right] \\
& \times\left\{\left[: \bar{\psi}(x / 2) \sigma_{\mu \nu} \psi(-x / 2):-: \bar{\psi}(-x / 2) \sigma_{\mu \nu} \psi(x / 2):\right]+g_{\mu \nu}[: \bar{\psi}(x / 2) \psi(-x / 2):+: \bar{\psi}(-x / 2) \psi(x / 2):]\right\} \\
& +: \bar{\psi}(x / 2) \gamma_{\mu} \psi(x / 2) \bar{\psi}(-x / 2) \gamma_{\nu} \psi(-x / 2):+\frac{2 x_{\mu} x_{\nu}-g_{\mu \nu} x^{2}}{\pi^{4}\left(x^{2}-i \epsilon\right)^{4}}
\end{aligned}
$$

The renormalization symbol $R$ is suppressed in the notation. The four-quark operator $\bar{\psi} \gamma_{\mu} \psi \bar{\psi} \gamma_{\nu} \psi$ has no singular coefficient on the light cone and is of minimal twist- 4 and the last term stems from disconnected diagrams. Both terms are therefore discarded, see Ref. [2].

The quark mass terms resulting from the mass-dependent part of the operator $(i \not b+m)$ are less singular and will also be omitted. In fact, the lowest twist contribution of all quark mass terms is contained in the operator

$$
m\left[: \bar{\psi}(x / 2) i \sigma_{\mu \nu} \psi(-x / 2):-: \bar{\psi}(-x / 2) i \sigma_{\mu \nu} \psi(x / 2):\right]
$$

with $\sigma_{\mu \nu}=(i / 2)\left[\gamma_{\mu}, \gamma_{\nu}\right]$ and is of twist-3. 
The first term of the expansion (2.2) is of main importance, because it contains the leading light-cone singularity and its minimal twist contribution is of twist-2. It also contains terms of higher twist (trace terms) and quark mass terms resulting from the mass dependence of the scalar propagator $\Delta(x \mathrm{~m})$. These terms are also less singular on the light cone.

Using the standard relations

$$
\begin{aligned}
\gamma_{\mu} \gamma_{\alpha} \gamma_{\nu} & =S_{\mu \alpha \nu \beta} \gamma^{\beta}+i \varepsilon_{\mu \alpha \nu \beta} \gamma^{5} \gamma^{\beta}, \\
S_{\mu \nu \mid \alpha \beta} & \equiv S_{\mu \alpha \nu \beta} \equiv\left(g_{\mu \alpha} g_{\nu \beta}+g_{\nu \alpha} g_{\mu \beta}-g_{\mu \nu} g_{\alpha \beta}\right),
\end{aligned}
$$

where we indicate that $S_{\mu \alpha \nu \beta}$ is symmetric in $\mu \nu$ and $\alpha \beta$, and noticing that the operator (2.2) is dominated by the lightcone singularity, $x^{2} \approx 0$, one arrives at

$$
\begin{aligned}
\hat{T}_{\mu \nu}(x) \approx & \frac{i \tilde{x}^{\alpha}}{\pi^{2}\left(x^{2}-i \epsilon\right)^{2}}\left\{S_{\mu \alpha \nu \beta} R T\left[O^{\beta}\left(\frac{\tilde{x}}{2},-\frac{\tilde{x}}{2}\right) \mathcal{S}\right]\right. \\
& \left.+i \varepsilon_{\mu \alpha \nu \beta} R T\left[O^{5 \beta}\left(\frac{\tilde{x}}{2},-\frac{\tilde{x}}{2}\right) \mathcal{S}\right]\right\},
\end{aligned}
$$

with the (unrenormalized) light-cone operators

$$
\begin{aligned}
O^{\beta}(\tilde{x} / 2,-\tilde{x} / 2) \equiv & \frac{i}{2}\left[: \bar{\psi}(\tilde{x} / 2) \gamma^{\beta} \psi(-\tilde{x} / 2):\right. \\
& \left.-: \bar{\psi}(-\tilde{x} / 2) \gamma^{\beta} \psi(\tilde{x} / 2):\right], \\
O^{5 \beta}(\tilde{x} / 2,-\tilde{x} / 2) \equiv & \frac{i}{2}\left[: \bar{\psi}(\tilde{x} / 2) \gamma^{5} \gamma^{\beta} \psi(-\tilde{x} / 2):\right. \\
& \left.+: \bar{\psi}(-\tilde{x} / 2) \gamma^{5} \gamma^{\beta} \psi(\tilde{x} / 2):\right] .
\end{aligned}
$$

This expansion has to be viewed as a simple form of the nonlocal light-cone expansion (1.10) from which the suitable $\Gamma$ structures can be read off. When considering general gauges the phase factors $U\left(\kappa_{1} \tilde{x}, \kappa_{2} \tilde{x}\right)$ must also be included into the light-cone operators $O^{\beta}$ and $O^{5 \beta}$. Taking into account also higher order terms of the $S$-matrix additional operator structures come into the play which, of course, are subleading.

The $\kappa$ integration appearing in the light-cone expansion (1.10) connects the coefficient functions, given here at the Born level,

$$
\begin{aligned}
C_{1 / 2}^{\prime}\left(x^{2}, \kappa_{1}, \kappa_{2}\right)= & \frac{i}{2 \pi^{2}\left(x^{2}-i \epsilon\right)^{2}}\left[\delta\left(\kappa_{1}-\frac{1}{2}\right) \delta\left(\kappa_{2}+\frac{1}{2}\right)\right. \\
& \left.\mp \delta\left(\kappa_{2}-\frac{1}{2}\right) \delta\left(\kappa_{1}+\frac{1}{2}\right)\right]
\end{aligned}
$$

with the respective operators. As is known from the general analysis of the light-cone expansion [3] the Fourier transforms of the coefficient functions in Eq. (1.10) are entire analytic functions with respect to the variables $\tilde{x} p_{i}$ which leads to a restriction of the integration range of $\kappa_{1}$ and $\kappa_{2}$ to the interval $[-1,+1]$.

For later convenience we introduce the variables $\kappa_{ \pm} \equiv \frac{1}{2}\left(\kappa_{2} \pm \kappa_{1}\right) \quad$ with $\quad \kappa_{1,2}=\kappa_{+} \mp \kappa_{-}$

and use the following integration measure in the light-cone expansion:

$$
\begin{aligned}
D^{2} \kappa \equiv & d \kappa_{1} d \kappa_{2} \theta\left(1-\kappa_{1}\right) \theta\left(1+\kappa_{1}\right) \theta\left(1-\kappa_{2}\right) \theta\left(1+\kappa_{2}\right) \\
= & 2 d \kappa_{+} d \kappa_{-} \theta\left(1-\kappa_{+}+\kappa_{-}\right) \theta\left(1+\kappa_{+}-\kappa_{-}\right) \theta \\
& \times\left(1-\kappa_{+}-\kappa_{-}\right) \theta\left(1+\kappa_{+}+\kappa_{-}\right) .
\end{aligned}
$$

In terms of the variables $\kappa_{+}$and $\kappa_{-}$the coefficient functions read

$$
\begin{aligned}
C_{1 / 2}\left(x^{2}, \kappa_{+}, \kappa_{-}\right)= & \frac{i}{4 \pi^{2}\left(x^{2}-i \epsilon\right)^{2}} \delta\left(\kappa_{+}\right) \\
& \times\left[\delta\left(\kappa_{-}+\frac{1}{2}\right) \mp \delta\left(\kappa_{-}-\frac{1}{2}\right)\right] .
\end{aligned}
$$

Using these conventions one obtains the following representation of the operator $\hat{T}_{\mu \nu}(x)$ :

$$
\begin{aligned}
R T\left[J_{\mu}\left(\frac{x}{2}\right) J_{\nu}\left(-\frac{x}{2}\right) \mathcal{S}\right] \\
\approx \int_{\mathbb{R}^{2}} D^{2} \kappa\left[C_{1}\left(x^{2}, \kappa_{+}, \kappa_{-}\right) S_{\mu \nu \mid} \alpha \beta \tilde{x}_{\alpha}\right. \\
\quad \times O_{\beta}\left(\left(\kappa_{+}-\kappa_{-}\right) \tilde{x},\left(\kappa_{+}+\kappa_{-}\right) \tilde{x}\right) \\
\quad-i C_{2}\left(x^{2}, \kappa_{+}, \kappa_{-}\right) \varepsilon_{\mu \nu}{ }^{\alpha \beta} \tilde{x}_{\alpha} \\
\left.\quad \times O_{\beta}^{5}\left(\left(\kappa_{+}-\kappa_{-}\right) \tilde{x},\left(\kappa_{+}-\kappa_{-}\right) \tilde{x}\right)\right] .
\end{aligned}
$$

In general, the renormalized nonlocal operators $O_{\beta}\left(\kappa_{1} \tilde{x}, \kappa_{2} \tilde{x}\right)$ and $O_{\beta}^{5}\left(\kappa_{1} \tilde{x}, \kappa_{2} \tilde{x}\right)$ containing the phase factors $U\left(\kappa_{1} \tilde{x}, \kappa_{2} \tilde{x}\right)$ are given by

$O_{\beta}\left(\kappa_{1} \tilde{x}, \kappa_{2} \tilde{x}\right)=\frac{i}{2} R T\left\{\left[: \bar{\psi}\left(\kappa_{1} \tilde{x}\right) \gamma_{\beta} U\left(\kappa_{1} \tilde{x}, \kappa_{2} \tilde{x}\right) \psi\left(\kappa_{2} \tilde{x}\right):\right.\right.$

$$
\left.\left.-: \bar{\psi}\left(\kappa_{2} \tilde{x}\right) \gamma_{\beta} U\left(\kappa_{1} \tilde{x}, \kappa_{2} \tilde{x}\right) \psi\left(\kappa_{1} \tilde{x}\right):\right] \mathcal{S}\right\},
$$

$$
\begin{aligned}
O_{\beta}^{5}\left(\kappa_{1} \tilde{x}, \kappa_{2} \tilde{x}\right)= & \frac{i}{2} R T\left\{\left[: \bar{\psi}\left(\kappa_{1} \tilde{x}\right) \gamma^{5} \gamma_{\beta} U\left(\kappa_{1} \tilde{x}, \kappa_{2} \tilde{x}\right) \psi\left(\kappa_{2} \tilde{x}\right):\right.\right. \\
& \left.\left.+: \bar{\psi}\left(\kappa_{2} \tilde{x}\right) \gamma^{5} \gamma_{\beta} U\left(\kappa_{1} \tilde{x}, \kappa_{2} \tilde{x}\right) \psi\left(\kappa_{1} \tilde{x}\right):\right] \mathcal{S}\right\}
\end{aligned}
$$

In the following we assume that the operators $O_{\beta}$ and $O_{\beta}^{5}$ are renormalized quantities.

\section{TWIST DECOMPOSITION AND MATRIX ELEMENTS}

The nonlocal operators $O_{\beta}\left(\kappa_{1} \tilde{x}, \kappa_{2} \tilde{x}\right)$ and $O_{\beta}^{5}\left(\kappa_{1} \tilde{x}, \kappa_{2} \tilde{x}\right)$, Eqs. (2.13) and (2.14), contain contributions of different 
twist. Here, the notion of twist is used in its original form [19] as

$$
\begin{aligned}
\text { (geometric) twist }(\tau)= & \text { scale dimension }(d) \\
& - \text { Lorentz } \operatorname{spin}(j) .
\end{aligned}
$$

The operators appearing in the expansion (2.12) of the Compton amplitude have to be decomposed into their various twist parts. On the light cone they contain contributions of twist-2, -3 , and -4

$$
\begin{aligned}
& O_{\beta}=O_{\beta}^{\mathrm{tw} 2}+O_{\beta}^{\mathrm{tw} 3}+O_{\beta}^{\mathrm{tw} 4}, \\
& O_{\beta}^{5}=O_{\beta}^{5 \mathrm{tw} 2}+O_{\beta}^{5 \mathrm{tw} 3}+O_{\beta}^{5 \mathrm{tw} 4} ;
\end{aligned}
$$

however, off the light cone they contain an infinite series of growing twist.

A group-theoretical procedure of the twist decomposition has been worked out in Refs. [17,20] and applied to various physically relevant light-ray operators. As a result, the twist-2 part of the operators (2.13) and (2.14) can be constructed out of the twist-2 (pseudo) scalar operators $O^{(5) \operatorname{tw} 2}\left(\kappa_{1} \tilde{x}, \kappa_{2} \tilde{x}\right)=\tilde{x}^{\beta} O_{\beta}^{(5)}\left(\kappa_{1} \tilde{x}, \kappa_{2} \tilde{x}\right)$ by applying the interior derivative

$$
d_{\beta}=(1+\tilde{x} \widetilde{\partial}) \widetilde{\partial}_{\beta}-\frac{1}{2} \tilde{x}_{\beta} \widetilde{\partial}^{2} \quad \text { with } \quad d^{2}=0
$$

on the undecomposed light-cone operators and performing a subsequent $\tau$ integration (which stems from the normalization of the local operators):

$$
\begin{aligned}
O_{\beta}^{\mathrm{tw} 2}\left(\kappa_{1} \tilde{x}, \kappa_{2} \tilde{x}\right) & =-\int_{0}^{1} d \tau \ln \tau d_{\beta} O\left(\kappa_{1} \tau \tilde{x}, \kappa_{2} \tau \tilde{x}\right) \\
& =\int_{0}^{1} d \tau\left[\tilde{\partial}_{\beta}+\frac{1}{2} \ln \tau \tilde{x}_{\beta} \widetilde{\partial}^{2}\right] O\left(\kappa_{1} \tau \tilde{x}, \kappa_{2} \tau \tilde{x}\right) .
\end{aligned}
$$

According to its structure the tracelessness of the operator (3.5) which corresponds on the light cone to the requirement $d^{\beta} O_{\beta}^{\mathrm{tw} 2}\left(\kappa_{1} \tilde{x}, \kappa_{2} \tilde{x}\right)=0$, is trivially fulfilled due to the property of $d^{\beta}$. An analogous relation holds for the axial vector and pseudoscalar operator. The twist-3 and twist-4 parts, however, cannot be constructed out of the (pseudo)scalar operator since the latter, when restricted to the light cone, is already of twist-2.

Since we want to extract the twist-2 part of the Compton amplitude, relation (3.5) will be applied to the matrix elements of the operators considered:

$$
\begin{aligned}
& \left\langle p_{2}, k\left|O_{\beta}^{\mathrm{tw} 2}\left(\kappa_{1} \tilde{x}, \kappa_{2} \tilde{x}\right)\right| p_{1}\right\rangle \\
& =\int_{0}^{1} d \tau\left[\tilde{\partial}_{\beta}+\frac{1}{2} \ln \tau \tilde{x}_{\beta} \tilde{\partial}^{2}\right]\left\langle p_{2}, k\left|O\left(\kappa_{1} \tau \tilde{x}, \kappa_{2} \tau \tilde{x}\right)\right| p_{1}\right\rangle .
\end{aligned}
$$

Here, the spins of the nucleons have been suppressed in the notation. Let us mention that the geometric twist decomposition of the matrix elements is due to the twist decomposition of the nonlocal operators. Usually, in phenomenological considerations another notion of twist, called "dynamical" twist, is considered which has been introduced in the decomposition of the (forward) matrix elements by Jaffe and $\mathrm{Ji}$ [21]. The interrelation of geometric and dynamic twist was considered in Ref. [22]. In the case of lowest twist-2 there appears no difference, but for higher twist the mismatch of dynamical twist with respect to geometric twist leads to differing structures.

Because of translation invariance,

$$
\begin{aligned}
& \left\langle p_{2}, k\left|O\left(\kappa_{1} \tilde{x}, \kappa_{2} \tilde{x}\right)\right| p_{1}\right\rangle \\
& \quad=e^{i \kappa_{+} \tilde{x} P_{-}}\left\langle p_{2}, k\left|O\left(-\kappa_{-} \tilde{x}, \kappa_{-} \tilde{x}\right)\right| p_{1}\right\rangle,
\end{aligned}
$$

it is more convenient to discuss the centered operator $O(-\kappa \tilde{x}, \kappa \tilde{x})$. Henceforth, for brevity, $\kappa_{-}$will be denoted by $\kappa$.

In the ordinary nonforward case one usually parametrizes the scalar matrix element by a Dirac- and a Pauli-type contribution,

$$
\begin{aligned}
& \mathcal{K}_{1}\left(\tilde{x}, p_{2}, p_{1}\right)=\tilde{x}^{\beta} \bar{u}\left(p_{2}\right) \gamma_{\beta} u\left(p_{1}\right), \\
& \mathcal{K}_{2}\left(\tilde{x}, p_{2}, p_{1}\right)=\tilde{x}^{\beta} \frac{1}{m_{0}} \bar{u}\left(p_{2}\right) \sigma_{\beta \alpha} p_{-}^{\alpha} u\left(p_{1}\right),
\end{aligned}
$$

where $u\left(p_{1}\right)$ and $\bar{u}\left(p_{2}\right)$ are on-shell spinors of the incoming and outgoing nucleons, $m_{0}$ is a dimensional mass scale which is kept fixed, and $\sigma_{\beta \alpha}=(i / 2)\left[\gamma_{\beta}, \gamma_{\alpha}\right]$. Because of $p_{-} \rightarrow 0$ the Pauli type factor $\mathcal{K}_{2}$ vanishes in the forward limit. Using these kinematic factors the matrix element can be parametrized as follows:

$$
\begin{aligned}
\left\langle p_{2}\right| & O(-\kappa \tilde{x}, \kappa \tilde{x})\left|p_{1}\right\rangle \\
& =\sum_{a=1}^{2} \mathcal{K}_{a}\left(\tilde{x}, p_{2}, p_{1}\right) \tilde{f}_{a}\left(\kappa \tilde{x} p_{+}, \kappa \tilde{x} p_{-}, p_{i} p_{j}, \mu^{2}\right) .
\end{aligned}
$$

Here, the coefficient functions $\widetilde{f}_{a}$ are the distribution amplitudes in $x$ space. They depend on $\kappa \tilde{x} p_{i}$ and all possible products of the two momenta, $p_{i} p_{j}=\left\{p_{1}^{2}, p_{2}^{2}, p_{1} p_{2}\right\}$, as well as on the renormalization scale $\mu$ and the coupling constant $g$.

If an outgoing meson is present in the process, which means that one has to construct the matrix elements $\left\langle p_{2}, k|O(-\kappa \tilde{x}, \kappa \tilde{x})| p_{1}\right\rangle$, the representation (3.10) of the scalar matrix element must be modified because of the presence of the additional momentum $k$. Especially, further kinematic structures occur. They can be determined in a straightforward way by using the following parametrization of the scalar matrix element:

$$
\left\langle p_{2}, k|O(-\kappa \tilde{x}, \kappa \tilde{x})| p_{1}\right\rangle=\bar{u}\left(p_{2}\right) \Lambda\left(\kappa \tilde{x}, p_{2}, k, p_{1}\right) u\left(p_{1}\right),
$$


with

$$
\Lambda\left(\kappa \tilde{x}, p_{2}, k, p_{1}\right)=\mathbf{A} I+\mathbf{B}_{\alpha} \gamma^{\alpha}+\mathbf{C}_{[\alpha \beta]} \sigma^{\alpha \beta}+\mathbf{D}_{\alpha} \gamma^{5} \gamma^{\alpha}
$$

It is an easy task to find the general structure of $\mathbf{A}-\mathbf{D}$ allowed by the momenta $p_{1 \alpha}, p_{2 \alpha}, k_{\alpha}$, the metric $g_{\alpha \beta}$ and the Levi-Civita tensor $\varepsilon_{\beta \alpha \gamma \delta}$, demanding $\Lambda$ not to be a pseudoscalar.

Using the equations of motion for the hadronic momenta $p_{1 \mu}$ and $p_{2 \mu}$ with $M$ denoting the nucleon mass,

$$
\begin{aligned}
& \gamma^{\alpha} p_{1 \alpha} u\left(p_{1}\right)=M u\left(p_{1}\right), \\
& \bar{u}\left(p_{2}\right) \gamma^{\alpha} p_{2 \alpha}=M \bar{u}\left(p_{2}\right),
\end{aligned}
$$

the decomposition of the matrix element (3.11) is

$$
\begin{aligned}
& \mathcal{K}_{1}^{\prime}=(\bar{u} u), \\
& \mathcal{K}_{2}^{\prime}=(\bar{u} \widetilde{x} u), \\
& \mathcal{K}_{3}^{\prime}=\frac{1}{m_{0}}(\bar{u} k u), \\
& \mathcal{K}_{4}^{\prime}=\frac{1}{m_{0}}(\bar{u} \widetilde{\mathcal{K}} k u), \\
& \mathcal{K}_{5}^{\prime}=\frac{1}{m_{0}^{3}} i \varepsilon_{\beta \alpha \gamma \delta} \tilde{x}^{\beta} p_{2}^{\alpha} k^{\gamma} p_{1}^{\delta}\left(\bar{u} \gamma^{5} u\right),
\end{aligned}
$$

where an auxiliary mass $m_{0}$ has been introduced in order to get kinematic structures of equal dimensionality. Then, the scalar matrix element is parametrized by a sum over the five kinematic factors $\mathcal{K}_{a}^{\prime}$ as follows:

$$
\left\langle p_{2}, k|O(-\kappa \tilde{x}, \kappa \tilde{x})| p_{1}\right\rangle=\sum_{a=1}^{5} \mathcal{K}_{a}^{\prime}(\tilde{x}, \mathbf{p}) \tilde{f}_{a}^{\prime}\left(\kappa \tilde{x} \mathbf{p}, \mathbf{p}_{i} \mathbf{p}_{j}, \mu^{2}\right)
$$

where $\mathbf{p} \equiv\left\{\mathbf{p}_{i}\right\}=\left\{p_{1}, p_{2}, k\right\}$ generically denotes the multivector in the space of all the (three) hadronic momenta.

Although one possible set of kinematic factors is given by Eq. (3.16), it will be more convenient to choose another one which is also linearly independent and contains the original kinematic factors $\mathcal{K}_{a}^{\prime}$. Mimicking the Dirac and Pauli structures we choose

$$
\begin{aligned}
& \mathcal{K}_{1}\left(\tilde{x}, p_{2}, k, p_{1}\right)=\tilde{x}^{\beta}\left(\bar{u} \gamma_{\beta} u\right), \\
& \mathcal{K}_{2}\left(\tilde{x}, p_{2}, k, p_{1}\right)=\tilde{x}^{\beta} \frac{1}{m_{0}}\left(\bar{u} \sigma_{\beta \alpha} P_{-}^{\alpha} u\right), \\
& \mathcal{K}_{3}\left(\tilde{x}, p_{2}, k, p_{1}\right)=\tilde{x}^{\beta} \frac{1}{m_{0}^{2}}\left(\bar{u} k_{\beta} \gamma_{\alpha} k^{\alpha} u\right),
\end{aligned}
$$

$$
\begin{aligned}
& \mathcal{K}_{4}\left(\tilde{x}, p_{2}, k, p_{1}\right)=\tilde{x}^{\beta} \frac{1}{m_{0}}\left(\bar{u} \sigma_{\beta \alpha} k^{\alpha} u\right), \\
& \mathcal{K}_{5}\left(\tilde{x}, p_{2}, k, p_{1}\right)=\tilde{x}^{\beta} \frac{1}{m_{0}^{3}} i \varepsilon_{\beta \alpha \gamma \delta} p_{2}^{\alpha} k^{\gamma} p_{1}^{\delta}\left(\bar{u} \gamma^{5} u\right)
\end{aligned}
$$

for the matrix element of the scalar operator and

$$
\begin{aligned}
& \mathcal{K}_{1}^{5}\left(\tilde{x}, p_{2}, k, p_{1}\right)=\tilde{x}^{\beta}\left(\bar{u} \gamma^{5} \gamma_{\beta} u\right), \\
& \mathcal{K}_{2}^{5}\left(\tilde{x}, p_{2}, k, p_{1}\right)=\tilde{x}^{\beta} \frac{1}{m_{0}}\left(\bar{u} \gamma^{5} \sigma_{\beta \alpha} P_{-}^{\alpha} u\right), \\
& \mathcal{K}_{3}^{5}\left(\tilde{x}, p_{2}, k, p_{1}\right)=\tilde{x}^{\beta} \frac{1}{m_{0}^{2}}\left(\bar{u} \gamma^{5} k_{\beta} \gamma_{\alpha} k^{\alpha} u\right), \\
& \mathcal{K}_{4}^{5}\left(\tilde{x}, p_{2}, k, p_{1}\right)=\tilde{x}^{\beta} \frac{1}{m_{0}}\left(\bar{u} \gamma^{5} \sigma_{\beta \alpha} k^{\alpha} u\right), \\
& \mathcal{K}_{5}^{5}\left(\tilde{x}, p_{2}, k, p_{1}\right)=\tilde{x}^{\beta} \frac{1}{m_{0}^{3}} i \varepsilon_{\beta \alpha \gamma \delta} p_{2}^{\alpha} k^{\gamma} p_{1}^{\delta}(\bar{u} u)
\end{aligned}
$$

for the matrix element of the pseudoscalar operator. For explicit calculations it is important to note that each factor $\mathcal{K}_{a}^{(5)}$ has a linear $\tilde{x}$ dependence,

$$
\begin{aligned}
& \mathcal{K}_{a}(\tilde{x}, \mathbf{p})=\tilde{x}^{\beta} \mathcal{K}_{a \beta}(\mathbf{p}), \\
& \mathcal{K}_{a}^{5}(\tilde{x}, \mathbf{p})=\tilde{x}^{\beta} \mathcal{K}_{a \beta}^{5}(\mathbf{p}) .
\end{aligned}
$$

Using the equations of motion again, the factors $\mathcal{K}_{a}$ equal the following combinations of $\mathcal{K}_{a}^{\prime}$ :

$$
\begin{aligned}
& \mathcal{K}_{1}=\mathcal{K}_{2}^{\prime}, \\
& \mathcal{K}_{2}=\frac{i}{m_{0}}\left(\tilde{x} p_{2}+\tilde{x} k+\tilde{x} p_{1}\right) \mathcal{K}_{1}^{\prime}-2 i \frac{M}{m_{0}} \mathcal{K}_{2}^{\prime}-i \mathcal{K}_{4}^{\prime}, \\
& \mathcal{K}_{3}=\frac{1}{m_{0}}(\tilde{x} k) \mathcal{K}_{3}^{\prime}, \\
& \mathcal{K}_{4}=\frac{i}{m_{0}}(\tilde{x} k) \mathcal{K}_{1}^{\prime}-i \mathcal{K}_{4}^{\prime}, \\
& \mathcal{K}_{5}=\mathcal{K}_{5}^{\prime} .
\end{aligned}
$$

This shows that the $\mathcal{K}_{a}$ constitute a suitable set of kinematic factors for the scalar matrix element. In the limit $k \rightarrow 0$ they obviously reduce to the Dirac and Pauli structures. Analogous statements hold for $\mathcal{K}_{a}^{5}$. 
The decomposition of the matrix element of $O(-\kappa \tilde{x}, \kappa \tilde{x})$ and $O^{5}(-\kappa \tilde{x}, \kappa \tilde{x})$ now reads

$$
\begin{aligned}
& \left\langle p_{2}, k|O(-\kappa \tilde{x}, \kappa \tilde{x})| p_{1}\right\rangle \\
& =\sum_{a=1}^{5} \mathcal{K}_{a}(\tilde{x}, \mathbf{p}) \tilde{f}_{a}\left(\kappa \tilde{x} \mathbf{p}, \mathbf{p}_{i} \mathbf{p}_{j}, \mu^{2}\right), \\
& \left\langle p_{2}, k\left|O^{5}(-\kappa \tilde{x}, \kappa \tilde{x})\right| p_{1}\right\rangle \\
& =\sum_{a=1}^{5} \mathcal{K}_{a}^{5}(\tilde{x}, \mathbf{p}) \tilde{f}_{a}^{5}\left(\kappa \tilde{x} \mathbf{p}, \mathbf{p}_{i} \mathbf{p}_{j}, \mu^{2}\right) .
\end{aligned}
$$

In principle there is also a dependence on variables like $\mathbf{p}_{i} \mathbf{p}_{j} x^{2}$. Because the latter dependence vanishes on the light cone, it will not be discussed in the further considerations. As has been shown in Ref. [18] the whole $x^{2}$ dependence is governed by harmonic extension off the light cone if the operator structure is already given on cone. The $\mu^{2}$ dependence is governed by the evolution equations obeyed by the functions $\tilde{f}_{a}$ and will be discussed in Sec. VI.

As a next step, a Fourier transformation of the functions $\widetilde{f}_{a}$ is performed,

$$
\tilde{f}_{a}(\kappa \tilde{x} \mathbf{p})=\int_{\mathbb{R}^{3}} D^{3} z e^{-i \kappa \tilde{x}(\mathbf{p z})} f_{a}(\mathbf{z})
$$

Because also the functions $\widetilde{f}_{a}(\kappa \tilde{x} \mathbf{p})$ are entire analytic in the variables $\kappa \tilde{x} \mathbf{p}$, the support of their Fourier transforms $f_{a}(\mathbf{z})$ is restricted to the interval $[-1,+1]$ in the variables $\mathbf{z}$ $=\left(z_{1}, z_{2}, z_{3}\right)$. Therefore, the measure

$$
\begin{aligned}
D^{3} z \equiv & d z_{1} d z_{2} d z_{3} \theta\left(1-z_{1}\right) \theta\left(1+z_{1}\right) \theta\left(1-z_{2}\right) \\
& \times \theta\left(1+z_{2}\right) \theta\left(1-z_{3}\right) \theta\left(1+z_{3}\right)
\end{aligned}
$$

has been introduced to realize this support. pz is simply the product of the vectors $\mathbf{p}$ and $\mathbf{z}$, see Eq. (3.24). To get a representation in the momenta $P_{ \pm}$and $k$ one introduces the variables

$$
z_{+}=\frac{1}{2}\left(z_{1}+z_{2}\right), \quad z_{-}=\frac{1}{2}\left(z_{2}-z_{1}\right), \quad z_{k}=z_{3}-z_{2},
$$

with

$$
z_{1}=z_{+}-z_{-}, \quad z_{2}=z_{+}+z_{-}, \quad z_{3}=z_{+}+z_{-}+z_{k} .
$$

Using the abbreviation

$$
\mathcal{P} \equiv P_{+} z_{+}+P_{-} z_{-}+k z_{k}=p_{1} z_{1}+p_{2} z_{2}+k z_{3} \equiv \mathbf{p z},
$$

the scalar matrix element is represented by

$$
\begin{aligned}
& \left\langle p_{2}, k|O(-\kappa \tilde{x}, \kappa \tilde{x})| p_{1}\right\rangle \\
& =\sum_{a=1}^{5} \mathcal{K}^{a}(\tilde{x}, \mathbf{p}) \int_{\mathbb{R}^{3}} D^{3} z e^{-i \kappa \tilde{x} \mathcal{P}} f_{a}\left(z_{+}, z_{-}, z_{k}\right) .
\end{aligned}
$$

In the following the explicit summation over $a$ will be omitted, but will be indicated by the position of the index $a$.

The expression (3.25) will be inserted into Eq. (3.6) to calculate the matrix element of $O_{\mu}^{\mathrm{tw} 2}$. Thereby, it is important to state that the $\tau$-scaling in equation (3.6) refers to $\kappa$ and not to $\tilde{x}$. Performing a change of variables $z_{ \pm} \rightarrow z_{ \pm} / \tau$ and $z_{k} \rightarrow z_{k} / \tau$ we get the following form of the matrix element, for the general case $\kappa_{1} \neq-\kappa_{2}$ :

$$
\begin{aligned}
\left\langle p_{2}, k\left|O_{\beta}^{\mathrm{tw}} 2\left(\kappa_{1} \tilde{x}, \kappa_{2} \tilde{x}\right)\right| p_{1}\right\rangle & =\int_{\mathbb{R}^{3}} d z_{+} d z_{-} d z_{k} \int_{0}^{1} d \tau\left[\tilde{\partial}_{\beta} \frac{1}{2} \ln \tau \tilde{x}_{\beta} \tilde{\partial}^{2}\right] \\
& \times \frac{1}{\tau^{3}} e^{i \tilde{x}\left(\kappa_{+} \tau P_{-}-\kappa \mathcal{P}\right)} \tilde{x}_{\rho} \mathcal{K}^{a \rho} \\
& \times(\mathbf{p}) f_{a}\left(\frac{z_{+}}{\tau}, \frac{z_{-}}{\tau}, \frac{z_{k}}{\tau}\right) \Theta\left(\tau, z_{+}, z_{-}, z_{k}\right),
\end{aligned}
$$

where the abbreviation

$$
\begin{aligned}
\Theta\left(\tau, z_{+}, z_{-}, z_{k}\right) \equiv & 2 \theta\left(\tau-z_{+}+z_{-}\right) \theta\left(\tau+z_{+}-z_{-}\right) \\
& \times \theta\left(\tau-z_{+}-z_{-}\right) \theta\left(\tau+z_{+}+z_{-}\right) \\
& \times \theta\left(\tau-z_{+}-z_{-}-z_{k}\right) \theta\left(\tau+z_{+}+z_{-}+z_{k}\right)
\end{aligned}
$$

has been used. Carrying out the differentiations we get

$$
\begin{aligned}
\left\langle p_{2}, k\right| & O_{\beta}^{\mathrm{tw} 2}\left(\kappa_{1} \tilde{x}, \kappa_{2} \tilde{x}\right)\left|p_{1}\right\rangle \\
= & \int_{\mathbb{R}^{3}} d z_{+} d z_{-} d z_{k} \int_{0}^{1} \frac{d \tau}{\tau^{3}} e^{i \tilde{x}\left(\kappa_{+} \tau P_{-}-\kappa \mathcal{P}\right)} \mathcal{K}^{a \rho}(\mathbf{p}) \\
& \times f_{a}\left(\frac{z_{+}}{\tau}, \frac{z_{-}}{\tau}, \frac{z_{k}}{\tau}\right) \Theta\left(\tau, z_{+}, z_{-}, z_{k}\right) \\
& \times\left[g_{\beta \rho}+i\left(\kappa_{+} \tau P_{-\beta}-\kappa \mathcal{P}_{\beta}\right) \tilde{x}_{\rho}-\frac{\tilde{x}_{\beta}}{2}\right. \\
& \left.\times \ln \tau\left[\left(\kappa_{+} \tau P_{-}-\kappa \mathcal{P}\right)^{2} \tilde{x}_{\rho}-2 i\left(\kappa_{+} \tau P_{-\rho}-\kappa \mathcal{P}_{\rho}\right)\right]\right]
\end{aligned}
$$

This form of the matrix element is yet rather complicated. Since only the centered operator is needed in the following considerations, we set $\kappa_{+}=0$. 
To derive a simpler representation for the matrix element, the $\tau$ integration will now be comprised into the functions $F_{a}$ and $F_{a}^{\mathrm{tr}}$, the latter denoting the trace part, defined by

$$
\begin{aligned}
& F_{a}(\mathbf{z}) \equiv F_{a}\left(z_{+}, z_{-}, z_{k}\right)=\int_{0}^{1} d \tau \frac{1}{\tau^{3}} \hat{f}_{a}\left(\frac{z_{+}}{\tau}, \frac{z_{-}}{\tau}, \frac{z_{k}}{\tau}\right), \\
& F_{a}^{\mathrm{tr}}(\mathbf{z}) \equiv F_{a}^{\mathrm{tr}}\left(z_{+}, z_{-}, z_{k}\right)=\int_{0}^{1} d \tau \frac{\ln \tau}{\tau^{3}} \hat{f}_{a}\left(\frac{z_{+}}{\tau}, \frac{z_{-}}{\tau}, \frac{z_{k}}{\tau}\right),
\end{aligned}
$$

with $\hat{f}_{a}$ given by

$$
\hat{f}_{a}\left(\frac{z_{+}}{\tau}, \frac{z_{-}}{\tau}, \frac{z_{k}}{\tau}\right) \equiv f_{a}\left(\frac{z_{+}}{\tau}, \frac{z_{-}}{\tau}, \frac{z_{k}}{\tau}\right) \Theta\left(\tau, z_{+}, z_{-}, z_{k}\right) .
$$

Obviously, these distribution amplitudes are not independent, and the restricted integration range in $z$ space finally is contained in the support properties of the distribution amplitudes $F_{a}\left(z_{+}, z_{-}, z_{k}\right)$ and $F_{a}^{\mathrm{tr}}\left(z_{+}, z_{-}, z_{k}\right)$.

After the substitution of $F_{a}$ and $F_{a}^{\mathrm{tr}}$ in Eq. (3.28) with $\kappa_{+}=0$ one obtains

$$
\begin{aligned}
& \left\langle p_{2}, k\left|O_{\beta}^{\mathrm{tw} 2}(-\kappa \tilde{x}, \kappa \tilde{x})\right| p_{1}\right\rangle \\
& =\int_{\mathrm{R}^{3}} d z_{+} d z_{-} d z_{k} e^{-i \kappa \tilde{x} \mathcal{P}}\left[\left(g_{\beta \rho}-i \kappa \mathcal{P}_{\beta} \tilde{x}_{\rho}\right) F_{a}(\mathbf{z})\right. \\
& \left.\quad-\frac{\tilde{x}_{\beta}}{2}\left(\kappa^{2} \mathcal{P}^{2} \tilde{x}_{\rho}+2 i \kappa \mathcal{P}_{\rho}\right) F_{a}^{\mathrm{tr}}(\mathbf{z})\right] \mathcal{K}^{a \rho}(\mathbf{p})
\end{aligned}
$$

for the matrix element of the twist-2 part of the vector operator $O_{\mu}$. The matrix element of the axial vector operator $O_{\mu}^{5 \text { tw } 2}$ possesses a similar representation

$$
\begin{aligned}
\left\langle p_{2}, k\right| & O_{\beta}^{5 \mathrm{tw} 2}(-\kappa \tilde{x}, \kappa \tilde{x})\left|p_{1}\right\rangle \\
= & \int_{\mathrm{R}^{3}} d z_{+} d z_{-} d z_{k} e^{-i \kappa \tilde{x} \mathcal{P}}\left[\left(g_{\beta \rho}-i \kappa \mathcal{P}_{\beta} \tilde{x}_{\rho}\right) F_{a}^{5}(\mathbf{z})\right. \\
& \left.\quad-\frac{\tilde{x}_{\beta}}{2}\left(\kappa^{2} \mathcal{P}^{2} \tilde{x}_{\rho}+2 i \kappa \mathcal{P}_{\rho}\right) F_{a}^{5 \operatorname{tr}}(\mathbf{z})\right] \mathcal{K}^{5 a \rho}(\mathbf{p}),
\end{aligned}
$$

with $F_{a}^{5}$ and $F_{a}^{5 \text { tr }}$ defined analogously to $F_{a}$ and $F_{a}^{\mathrm{tr}}$, by exchanging $\hat{f}_{a}$ and $\hat{f}_{a}^{5}$ in Eqs. (3.29) and (3.30).

Here, some general remarks are in order. First, the triplevalued distribution amplitudes $F_{a}^{(5)}\left(z_{+}, z_{-}, z_{k}\right)$ and $F_{a}^{(5) \operatorname{tr}}\left(z_{+}, z_{-}, z_{k}\right)$ are uniquely related to the twist-2 (axial) vector operators and, in principle, should have been marked by the related twist $\tau=2$. However, since we do not consider higher twist this has been omitted. Second, every distribution amplitude of definite twist - also off the light cone-depends only on the $\mathbf{z}$ variables and, possibly, on the momenta $\mathbf{p}$. The $x$ dependence is completely contained in the accompanying factors including, of course, the exponential $e^{-i \kappa x \mathcal{P}}$. These general expressions which are given in terms of Bessel functions of the argument $(\kappa / 2) \sqrt{(x \mathcal{P})^{2}-x^{2} \mathcal{P}^{2}}$ have been determined in Ref. [18]. (For the twist-2 case of DIS this statement has already been made in Ref. [23].) Restricting onto the light-cone leads to the expressions (3.32) and (3.33). Third, these properties hold for the operators of definite twist and are transposed to the corresponding matrix elements, independently of how many particles (momenta) occur in the incoming and outgoing states. Therefore, if a Fourier transformation containing these matrix elements has to be performed this can be done by simply replacing $\tilde{x} \rightarrow x$. (In Appendix A we give these expressions explicitly together with their restriction onto the light cone.) This will be applied in the next section for the expressions Eqs. (3.32) and (3.33).

\section{COMPTON AMPLITUDE}

Now, we are in a position to compute the twist-2 part of the Compton amplitude (1.3). Of course, we need it in the extended Bjorken region and, therefore, can restrict our consideration to the neighborhood of the light cone. Thus, we will take the matrix elements (3.32) and (3.33) with $\tilde{x}$ replaced by $x$. Merging everything together, we use the representation (2.12) for the time-ordered product of two hadronic currents, insert Eqs. (3.32) and (3.33) for the matrix elements of $O_{\beta}^{\mathrm{tw} 2}$ and $O_{\beta}^{5 \mathrm{tw} 2}$ and obtain

$$
\begin{aligned}
& T_{\mu \nu}^{\mathrm{tw} 2}\left(p_{2}, k, p_{1}, q\right) \\
& =i \int d^{4} x e^{i q x} \int_{\mathbb{R}^{2}} D^{2} \kappa x_{\alpha}\left[C_{1}\left(x^{2}, \kappa_{+}, \kappa\right) S_{\mu \nu \mid} \alpha \beta\right. \\
& \quad \times\left\langle p_{2}, k\left|O_{\beta}^{\mathrm{tw} 2}\left(\left(\kappa_{+}-\kappa\right) x,\left(\kappa_{+}+\kappa\right) x\right)\right| p_{1}\right\rangle \\
& \quad-i C_{2}\left(x^{2}, \kappa_{+}, \kappa\right) \varepsilon_{\mu \nu} \alpha \beta \\
& \left.\quad \times\left\langle p_{2}, k\left|O_{\beta}^{5 \mathrm{tw} 2}\left(\left(\kappa_{+}-\kappa\right) x,\left(\kappa_{+}+\kappa\right) x\right)\right| p_{1}\right\rangle\right] .
\end{aligned}
$$

The arguments $\kappa_{+}$and $\kappa$ of the coefficient functions $C_{i}\left(x^{2}, \kappa_{+}, \kappa\right)$ are fixed at $\kappa_{+}=0$ and $\kappa= \pm 1 / 2$, so that we can use the symmetry properties of the operators $O_{\beta}$ and $O_{\beta}^{5}$,

$$
\begin{aligned}
& O_{\beta}(-\kappa x, \kappa x)=-O_{\beta}(\kappa x,-\kappa x), \\
& O_{\beta}^{5}(-\kappa x, \kappa x)=O_{\beta}^{5}(\kappa x,-\kappa x) .
\end{aligned}
$$


For the computation of the Compton amplitude it suffices to know the operators at these given points, but for the investigation of the evolution of the matrix elements their representation at general values of $\kappa_{+}$and $\kappa$ is needed, see Sec. VI for the details.

Performing the $\kappa$ integration one obtains

$$
\begin{aligned}
T_{\mu \nu}^{\mathrm{tw} 2}\left(p_{2}, k, p_{1}, q\right)= & -2 \int_{\mathbb{R}^{3}} d z_{+} d z_{-} d z_{k} \int \frac{d^{4} x}{2 \pi^{2}} e^{i \mathcal{Q} x} \\
& \times \frac{x_{\alpha}}{\left(x^{2}-i \epsilon\right)^{2}}\left\{S _ { \mu \nu | } { } ^ { \alpha \beta } \left[\left(g_{\beta \rho}-\frac{i}{2} \mathcal{P}_{\beta} x_{\rho}\right) F_{a}\right.\right. \\
& \left.-x_{\beta}\left(\frac{1}{8} \mathcal{P}^{2} x_{\rho}+\frac{i}{2} \mathcal{P}_{\rho}\right) F_{a}^{\mathrm{tr}}\right] \mathcal{K}^{a \rho}-i \varepsilon_{\mu \nu}{ }^{\alpha \beta} \\
& \left.\times\left(g_{\beta \rho}-\frac{i}{2} \mathcal{P}_{\beta} x_{\rho}\right) F_{a}^{5} \mathcal{K}^{5 a \rho}\right\},
\end{aligned}
$$

where the abbreviation

$$
\mathcal{Q} \equiv q-\frac{1}{2} \mathcal{P}=q-\frac{1}{2}\left(P_{+} z_{+}+P_{-} z_{-}+k z_{k}\right)
$$

has been used. The $x_{\beta}$ term which results from the traces of the twist-2 operator vanishes for the axial matrix element because $x_{\alpha} x_{\beta}$ is symmetric. As a last step in the computation of the Compton amplitude the Fourier transformation is carried out by using

$\int \frac{d^{4} x}{2 \pi^{2}} e^{i \mathcal{Q} x} \frac{x_{\alpha}}{\left(x^{2}-i \epsilon\right)^{2}}=\frac{\mathcal{Q}_{\alpha}}{\mathcal{Q}^{2}+i \epsilon}$,

$\int \frac{d^{4} x}{2 \pi^{2}} e^{i \mathcal{Q} x} \frac{x_{\alpha} x_{\beta}}{\left(x^{2}-i \epsilon\right)^{2}}=-i \frac{g_{\alpha \beta}}{\mathcal{Q}^{2}+i \epsilon}+2 i \frac{\mathcal{Q}_{\alpha} \mathcal{Q}_{\beta}}{\left(\mathcal{Q}^{2}+i \epsilon\right)^{2}}$,

$\int \frac{d^{4} x}{2 \pi^{2}} e^{i \mathcal{Q} x} \frac{x_{\alpha} x_{\beta} x_{\rho}}{\left(x^{2}-i \epsilon\right)^{2}}=2 \frac{g_{\alpha \beta} \mathcal{Q}_{\rho}+g_{\beta \rho} \mathcal{Q}_{\alpha}+g_{\alpha \rho} \mathcal{Q}_{\beta}}{\left(\mathcal{Q}^{2}+i \epsilon\right)^{2}}$ $-8 \frac{\mathcal{Q}_{\alpha} \mathcal{Q}_{\beta} \mathcal{Q}_{\rho}}{\left(\mathcal{Q}^{2}+i \epsilon\right)^{3}}$,

and the summation over $\alpha$ and $\beta$ is performed in the symmetric part of $T_{\mu \nu}^{\mathrm{tw} 2}$ by using the form (2.5) for $S_{\mu \nu \mid}{ }^{\alpha \beta}$. Then for the Compton amplitude we get the result

$$
\begin{aligned}
T_{\mu \nu}^{\mathrm{tw} 2}= & -2 \int_{\mathbb{R}^{3}} d z_{+} d z_{-} d z_{k} \frac{1}{\mathcal{Q}^{2}+i \epsilon} \\
& \times\left(-i \varepsilon_{\mu \nu}{ }^{\alpha \beta} q_{\alpha}\left\{g_{\beta \rho}+\frac{\mathcal{P}_{\beta} \mathcal{Q}_{\rho}}{\mathcal{Q}^{2}+i \epsilon}\right\} F_{a}^{5} \mathcal{K}^{5 a \rho}\right. \\
& +\left\{g_{\mu \rho}\left(q_{\nu}-\mathcal{P}_{\nu}\right)+g_{\nu \rho}\left(q_{\mu}-\mathcal{P}_{\mu}\right)-g_{\mu \nu}\left(q_{\rho}-\mathcal{P}_{\rho}\right)\right. \\
& \left.+\frac{\mathcal{Q}_{\rho}}{\mathcal{Q}^{2}+i \epsilon}\left[\mathcal{Q}_{\mu} \mathcal{P}_{\nu}+\mathcal{Q}_{\nu} \mathcal{P}_{\mu}-g_{\mu \nu} \mathcal{P} \mathcal{Q}\right]\right\} F_{a} \mathcal{K}^{a \rho} \\
& +\left\{g_{\mu \nu} \mathcal{P}_{\rho}+\frac{1}{\mathcal{Q}^{2}+i \epsilon}\left[\left(2 \mathcal{Q}_{\mu} \mathcal{Q}_{\nu}-g_{\mu \nu} \mathcal{Q}^{2}\right) \mathcal{P}_{\rho}\right.\right. \\
& \left.-\frac{1}{2}\left(g_{\nu \rho} \mathcal{Q}_{\mu}+g_{\mu \rho} \mathcal{Q}_{\nu}-2 g_{\mu \nu} \mathcal{Q}_{\rho}\right) \mathcal{P}^{2}\right] \\
& \left.\left.+\frac{\mathcal{Q}_{\rho} \mathcal{P}^{2}}{\left(\mathcal{Q}^{2}+i \epsilon\right)^{2}}\left(2 \mathcal{Q}_{\mu} \mathcal{Q}_{\nu}-g_{\mu \nu} \mathcal{Q}^{2}\right)\right\} F_{a}^{\mathrm{tr}} \mathcal{K}^{a \rho}\right),
\end{aligned}
$$

which is expanded with respect to the functions $F_{a}^{5}, F_{a}$, and $F_{a}^{\mathrm{tr}}$ with the trace term separated from the antisymmetric and remaining symmetric part.

This structure of the Compton amplitude is a generic one because it is also valid for the ordinary nonforward case: The additional structures arising due to the momentum $k$ are hidden in the summation over the structure functions $F_{a}, F_{a}^{5}$, and $F_{a}^{\mathrm{tr}}$ and in the definitions of $\mathcal{P}$ and $\mathcal{Q}$. The reason for that result is an outcome of the twist structure of the operator which is the same for all the matrix elements under consideration. It holds also for the case of $n$ outgoing mesons.

For $\mathcal{P}=p_{+} z_{+}+p_{-} z_{-}$and summation over the Dirac and Pauli structures one reproduces the form of the Compton amplitude given in Ref. [7]. Here, the additional terms containing the functions $F_{a}^{\mathrm{tr}}$ arise, because the trace term in Eq. (3.6) has been taken into account. If $k$ is not present in the process this term vanishes in the limit

$$
\begin{aligned}
p_{i} p_{j} & \approx 0, \\
\gamma^{\mu} p_{1 \mu} u\left(p_{1}\right) & \approx 0, \\
\bar{u}\left(p_{2}\right) \gamma^{\mu} p_{2 \mu} & \approx 0,
\end{aligned}
$$

setting $M$ and $t=\left(p_{2}-p_{1}\right)^{2}$ to zero. Therefore these terms have been omitted in Ref. [7]. If the five kinematic factors $\mathcal{K}_{a}(x, \mathbf{p})$ containing the momentum $k$ are present this is $a$ priori no longer the case since nonvanishing contractions like $(\bar{u} k u)$ appear. Therefore, this term has been taken into full account here.

In general, terms containing the product $\mathcal{P}_{\rho} \mathcal{K}^{a \rho}$ do not vanish in the massless limit, while terms containing $\mathcal{P}^{2}, \mathbf{p}_{i} \mathbf{p}_{j}$, are small compared to the large invariants. This leads to the approximation 


$$
\frac{1}{\mathcal{Q}^{2}+i \epsilon} \approx \frac{1}{q^{2}-q \mathcal{P}+i \epsilon}=-\frac{1}{q P_{+}} \frac{1}{\xi+\left(z_{+}+\eta z_{-}+\chi z_{k}\right)-i \epsilon} .
$$

We apply these approximations to the Compton amplitude and get a representation for its twist- 2 part in the massless limit and in the extended Bjorken region, which depends explicitly on the three variables $z_{ \pm}$and $z_{k}$ :

$$
\begin{aligned}
T_{\mu \nu}^{\mathrm{tw} 2}= & -2 \int_{\mathbb{R}^{3}} d z_{+} d z_{-} d z_{k}\left[-\frac{1}{q P_{+}} \frac{1}{\xi+\left(z_{+}+\eta z_{-}+\chi z_{k}\right)-i \epsilon}\left\{\left[g_{\mu \rho}\left(q_{\nu}-\mathcal{P}_{\nu}\right)+g_{\nu \rho}\left(q_{\mu}-\mathcal{P}_{\mu}\right)\right.\right.\right. \\
& \left.\left.-g_{\mu \nu}\left(q_{\rho}-\mathcal{P}_{\rho}\right)\right] \cdot F_{a}\left(z_{+}, z_{-}, z_{k}\right) \mathcal{K}^{a \rho}+g_{\mu \nu} \mathcal{P}_{\rho} \cdot F_{a}^{\mathrm{tr}}\left(z_{+}, z_{-}, z_{k}\right) \mathcal{K}^{a \rho}-i \varepsilon_{\mu \nu}{ }^{\alpha \beta} q_{\alpha} g_{\beta \rho} \cdot F_{a}^{5}\left(z_{+}, z_{-}, z_{k}\right) \mathcal{K}^{5 a \rho}\right\} \\
& +\frac{1}{\left(q P_{+}\right)^{2}} \cdot \frac{1}{\left[\xi+\left(z_{+}+\eta z_{-}+\chi z_{k}\right)-i \epsilon\right]^{2}}\left\{\left[q_{\mu} \mathcal{P}_{\nu}+q_{\nu} \mathcal{P}_{\mu}-\mathcal{P}_{\mu} \mathcal{P}_{\nu}-g_{\mu \nu} q \cdot \mathcal{P}\right] \mathcal{Q}_{\rho} \cdot F_{a}\left(z_{+}, z_{-}, z_{k}\right) \mathcal{K}^{a \rho}\right. \\
& \left.\left.+\left(2 \mathcal{Q}_{\mu} \mathcal{Q}_{\nu}-g_{\mu \nu} q^{2}+g_{\mu \nu} q \cdot \mathcal{P}\right) \mathcal{P}_{\rho} \cdot F_{a}^{\mathrm{tr}}\left(z_{+}, z_{-}, z_{k}\right) \mathcal{K}^{a \rho}-i \varepsilon_{\mu \nu}{ }^{\alpha \beta} q_{\alpha} \mathcal{P}_{\beta} \mathcal{Q}_{\rho} \cdot F_{a}^{5}\left(z_{+}, z_{-}, z_{k}\right) \mathcal{K}^{5 a \rho}\right\}\right]
\end{aligned}
$$

This form of the Compton amplitude will be used in the further considerations.

\section{INTEGRAL RELATIONS}

The variables $z_{ \pm}$and $z_{k}$ are not directly measurable because they appear as Fourier variables of the distribution amplitudes $f_{a}$. The scaling variable $\xi$, however, can be regarded as a physical quantity and it is therefore quite natural to use the new variable

$$
t \equiv z_{+}+\eta z_{-}+\chi z_{k}
$$

as integration variable in the denominators of Eq. (4.10).

By the definition (3.24) the vector $\mathcal{P}$ contains the variables $z_{ \pm}$and $z_{k}$ and must be rewritten using the set $\left\{t, z_{-}, z_{k}\right\}$. This is simply done by introducing the combinations

$$
\begin{aligned}
& \pi \equiv P_{-}-\eta P_{+}, \\
& \tilde{\pi} \equiv k-\chi P_{+},
\end{aligned}
$$

which leads to the representation

$$
\mathcal{P}=P_{+} t+\pi z_{-}+\tilde{\pi} z_{k}
$$

The 4-vectors $\pi$ and $\tilde{\pi}$ define off-collinear directions with respect to the direction $P_{+}$; vectors along this momentum are denoted as collinear. Note that these vectors are nonforward still, since $\eta \neq 0$. $\mathcal{P}$ contains collinear and off-collinear contributions, the former of which are associated with the scaling variable $t$ only. It will turn out that these collinear parts play the dominant role in the process considered.

Different powers of $\mathcal{P}$ contained in Eq. (4.10), of course, will lead to a whole series of structure functions corresponding to different moments in $z_{-}$and $z_{k}$. Therefore, let us generally define double moments of the triple-valued distribution amplitudes leading to single-valued distributions as follows:

$$
\begin{aligned}
\hat{F}_{n_{1} n_{2}}^{a}(t ; \eta, \chi) \equiv & \frac{1}{t^{n_{1}+n_{2}}} \int d z_{-} \int d z_{k} z_{-}^{n_{1}} z_{k}^{n_{2}} F^{a} \\
& \times\left(t-\eta z_{-}-\chi z_{k}, z_{-}, z_{k}\right) \\
= & \frac{1}{t^{n_{1}+n_{2}}} \int_{0}^{1} \frac{d \tau}{\tau} \tau^{n_{1}+n_{2}} \cdot \hat{f}_{n_{1} n_{2}}^{a}\left(\frac{t}{\tau} ; \eta, \chi\right) \\
= & \int_{t}^{\operatorname{sign}(t)} \frac{d \lambda}{\lambda} \lambda^{-n_{1}-n_{2} \cdot \hat{f}_{n_{1} n_{2}}^{a}(\lambda ; \eta, \chi)}
\end{aligned}
$$

with

$$
\begin{aligned}
\hat{f}_{n_{1} n_{2}}^{a}\left(\frac{t}{\tau} ; \eta, \chi\right) \equiv & \int d z_{-} \int d z_{k} z_{-}^{n_{1}} z_{k}^{n_{2}} \\
& \times \hat{f}^{a}\left(\frac{t}{\tau}-\eta z_{-}-\chi z_{k}, z_{-}, z_{k}\right) .
\end{aligned}
$$

In fact, the values $n_{i}=0,1$ occur in the antisymmetric part and the values $n_{i}=0,1,2$ in the symmetric part. To keep the discussion short, we consider the antisymmetric part of the Compton amplitude in full detail and give the result for the symmetric part only for the leading terms, i.e., suppressing the trace terms. The explicit calculation shows that the latter terms do not contribute to the leading order in $\nu$.

Because the contractions $\mathcal{P}_{\rho} \mathcal{K}^{a \rho}$ are only present for the Dirac structure, $\mathcal{K}^{1 \rho}=\left(\bar{u} \gamma^{\rho} u\right)$, we discuss this term separately. Then, the antisymmetric part of Eq. (4.10) is given by 


$$
\begin{aligned}
T_{[\mu \nu]}^{\mathrm{tw} 2} \approx & 2 i \varepsilon_{\mu \nu}{ }^{\alpha \beta} \frac{q_{\alpha}}{q P_{+}} \int_{\mathrm{R}^{3}} d z_{+} d z_{-} d z_{k} \\
& \times\left[\frac{g_{\beta \rho}}{\xi+t-i \epsilon}-\frac{q_{\rho}}{q P_{+}} \frac{\mathcal{P}_{\beta}}{(\xi+t-i \epsilon)^{2}}\right] \\
& \times F_{a}^{5}\left(z_{+}, z_{-}, z_{k}\right) \mathcal{K}^{5 a \rho}(\mathbf{p}) \\
= & 2 i \varepsilon_{\mu \nu}{ }^{\alpha \beta} \frac{q_{\alpha}}{q P_{+}} \int_{-1}^{1} d t\left[\frac{g_{\beta \rho} \hat{F}_{00}^{5 a}(t)}{\xi+t-i \epsilon}-\frac{q_{\rho}}{q P_{+}}\right. \\
& \left.\times \frac{t\left[P_{+\beta} \hat{F}_{00}^{5 a}(t)+\pi_{\beta} \hat{F}_{10}^{5 a}(t)+\tilde{\pi}_{\beta} \hat{F}_{01}^{5 a}(t)\right]}{(\xi+t-i \epsilon)^{2}}\right] \mathcal{K}^{5 a \rho}(\mathbf{p}) .
\end{aligned}
$$

At this point it is necessary to perform a partial integration in the second term proportional to $q_{\rho}$ using the formula

$$
\begin{gathered}
\int_{-1}^{1} d t \frac{t^{m}}{(\xi+t-i \epsilon)^{2}} \cdot \hat{F}_{n_{1} n_{2}}^{5 a}(t ; \eta, \chi) \\
=\int_{-1}^{1} d t \frac{t^{m-1}}{\xi+t-i \epsilon}\left[m \cdot \hat{F}_{n_{1} n_{2}}^{5 a}(t ; \eta, \chi)\right. \\
-t^{\left.-n_{1}-n_{2} \cdot \hat{f}_{n_{1} n_{2}}^{5 a}(t ; \eta, \chi)\right],}
\end{gathered}
$$

which leads to

$$
\begin{aligned}
T_{[\mu \nu]}^{\mathrm{tw} 2} \approx & 2 i \varepsilon_{\mu \nu} \alpha \beta \frac{q_{\alpha}}{q P_{+}} \int_{-1}^{1} d t \frac{1}{\xi+t-i \epsilon} \\
& \times\left[g_{\beta \rho} \hat{F}_{00}^{5 a}-\frac{q_{\rho}}{q P_{+}}\left\{P_{+\beta}\left(\hat{F}_{00}^{5 a}-\hat{f}_{00}^{5 a}\right)\right.\right. \\
& \left.\left.+\pi_{\beta}\left(\hat{F}_{10}^{5 a}-\frac{\hat{f}_{10}^{5 a}}{t}\right)+\tilde{\pi}_{\beta}\left(\hat{F}_{01}^{5 a}-\frac{\hat{f}_{01}^{5 a}}{t}\right)\right\}\right] \mathcal{K}^{5 a \rho} .
\end{aligned}
$$

Several tensor structures contribute. Note that in the foregoing discussion no assumption has been made on the direction of the nucleon spin. As the polarization of the initial state nucleons in experiment is performed in outer magnetic fields, the direction of the nucleon spin is not related to other vectors in the system except for the condition $S_{i} p_{i}=0$ to hold.

The form (5.10) of the polarized Compton amplitude is very interesting because it includes a Wandzura-Wilczek (WW) like relation between the distribution amplitudes being associated to two of the tensor structures [24]. This relation becomes obvious, once the definitions

$$
\mathrm{G}_{1}^{a}(t ; \eta, \chi) \equiv \hat{f}_{00}^{5 a}(t ; \eta, \chi),
$$

$$
\begin{aligned}
& \mathrm{G}_{2}^{a}(t ; \eta, \chi) \equiv-\hat{f}_{00}^{5 a}(t ; \eta, \chi)+\int_{t}^{\operatorname{sign}(t)} \frac{d \lambda}{\lambda} \hat{f}_{00}^{5 a}(\lambda ; \eta, \chi), \\
& \mathrm{G}_{3}^{a}(t ; \eta, \chi) \equiv-\frac{\hat{f}_{10}^{5 a}(t ; \eta, \chi)}{t}+\int_{t}^{\operatorname{sign}(t)} \frac{d \lambda}{\lambda^{2}} \hat{f}_{10}^{5 a}(\lambda ; \eta, \chi), \\
& \mathrm{G}_{4}^{a}(t ; \eta, \chi) \equiv-\frac{\hat{f}_{01}^{5 a}(t ; \eta, \chi)}{t}+\int_{t}^{\operatorname{sign}(t)} \frac{d \lambda}{\lambda^{2}} \hat{f}_{01}^{5 a}(\lambda ; \eta, \chi)
\end{aligned}
$$

are made. This leads to a very simple form of the antisymmetric part of the Compton amplitude, namely,

$$
\begin{aligned}
T_{[\mu \nu]}^{\mathrm{tw} 2}= & 2 i \epsilon_{\mu \nu}{ }^{\alpha \beta} \frac{q_{\alpha}}{q P_{+}} \int_{-1}^{1} d t \frac{1}{\xi+t-i \epsilon}\left[g_{\beta \rho}\left(\mathrm{G}_{1}^{a}+\mathrm{G}_{2}^{a}\right)\right. \\
& \left.-\frac{q_{\rho}}{q P_{+}}\left(P_{+\beta} \mathrm{G}_{2}^{a}+\pi_{\beta} \mathrm{G}_{3}^{a}+\tilde{\pi}_{\beta} \mathrm{G}_{4}^{a}\right)\right] \mathcal{K}^{5 a \rho} .
\end{aligned}
$$

All the above functions $\mathrm{G}_{k}^{a}$ are expectation values of twist-2 operators. By definition $\mathrm{G}_{1}^{a}$ and $\mathrm{G}_{2}^{a}$ obey the following integral relation:

$$
\mathrm{G}_{2}^{a}(t ; \eta, \chi)=-\mathrm{G}_{1}^{a}(t ; \eta, \chi)+\int_{t}^{\operatorname{sign}(t)} \frac{d \lambda}{\lambda} \mathrm{G}_{1}^{a}(\lambda ; \eta, \chi)
$$

This relation between two twist- 2 quantities can be viewed as a generalization of the WW relation known from forward scattering $[25,26]$. It is a property of the collinear part of the polarized Compton amplitude $T_{[\mu \nu]}^{\mathrm{tw} 2}$ and connected to the 00 moment in $z_{-}$and $z_{k}$. The off-collinear vectors $\pi$ and $\tilde{\pi}$ are connected to the new parton distributions $\mathrm{G}_{3}^{a}$ and $\mathrm{G}_{4}^{a}$, which obey an integral representation in terms of the functions $\hat{f}_{10}^{5 a}$ and $\hat{f}_{01}^{5 a}$, respectively, similar to $\mathbf{G}_{2}^{a}$. However, unlike the case for $G_{1}$ the respective functions do not appear in the Compton amplitude. Therefore we obtain at the present level only one Wandzura-Wilczek-like relation between the twisttwo parts of the respective amplitudes. Of course all the functions $G_{i}$ do receive higher twist contributions, which were not discussed in the present paper. Also these contributions as emerging in the different amplitudes may obey similar integral relations. The generalization (5.15) of the WW relation has been obtained in Ref. [7] for the ordinary nonforward process (1.1) without outgoing meson. The foregoing discussion shows that it remains valid for the more general process (1.2).

The Wandzura-Wilczek relation Eq. (5.15) is an identity between physical amplitudes which emerges at the level of geometric twist-2 as a consequence of the fact that the distribution $\hat{f}_{00}^{5 a}(t, \eta ; \chi)$ appears two times in the decomposition of the Compton amplitude. These relations which determine 
the (geometric) twist-2 content of the dynamical twist-3 distributions have to be called geometric WW relations $[7,27-$ 30]. They are obtained by using solely group theoretical means lying behind the definition of (genuine) geometric twist, Eq. (3.1). These WW relations have to be distinguished from the so-called dynamical WW relations being obtained by using the QCD equations of motion as has been done by [31]. To bring it to the point: Geometric WW relations are written for distributions having equal geometric twist, whereas dynamic WW relations are written between distributions of equal dynamic twist. Despite having the same formal structure their physical content is different. For a detailed discussion of these relations in the case of meson wave functions see Ref. [29] which, however, with appropriate modifications also holds for general nonforward amplitudes (see also Ref. [20] where the case of quark distributions is discussed).

We now return to the discussion of the term containing the contractions $\mathcal{P}_{\rho} \mathcal{K}^{a \rho}$. To begin with, we first note that only the Dirac structure $\mathcal{K}^{1 \rho}$ is of relevance in this case and one may use the approximation

$$
\mathcal{P}_{\rho} \mathcal{K}^{(5) 1 \rho} \approx k_{\rho}\left[t+(1-\eta) z_{-}+(1-\chi) z_{k}\right] \mathcal{K}^{(5) 1 \rho}
$$

in the limit of vanishing nucleon masses. Even more general, one can state that the product $q_{\rho} \mathcal{K}^{(5) 1 \rho}$ is of order $\nu$ and $\mathcal{P}_{\rho} \mathcal{K}^{(5) a \rho}$ is of order $\mu$ with $\mu \ll \nu$. The additional terms are therefore of the nonleading type, but are interesting because these structures arise due to the meson momentum $k$.

Inserting the above approximation and performing again partial integrations leads to the complete form of $T_{[\mu \nu]}^{\mathrm{tw} 2}$.

$$
\begin{aligned}
T_{[\mu \nu]}^{\mathrm{tw} 2}= & 2 i \varepsilon_{\mu \nu}{ }^{\alpha \beta} \frac{q_{\alpha}}{q P_{+}} \int_{-1}^{1} d t \frac{1}{\xi+t-i \epsilon}\left\{\left[g_{\beta \rho}\left(\mathrm{G}_{1}^{a}+\mathrm{G}_{2}^{a}\right)\right.\right. \\
& \left.-\frac{q_{\rho}}{q P_{+}}\left(P_{+\beta} \mathrm{G}_{2}^{a}+\pi_{\beta} \mathrm{G}_{3}^{a}+\tilde{\pi}_{\beta} \mathrm{G}_{4}^{a}\right)\right] \mathcal{K}^{5 a \rho} . \\
& \left.+\left[\frac{k_{\rho}}{q P_{+}}\left(P_{+\beta} \mathrm{G}_{2}^{\prime}+\pi_{\beta} \mathrm{G}_{3}^{\prime}+\tilde{\pi}_{\beta} \mathrm{G}_{4}^{\prime}\right)\right] \mathcal{K}^{51 \rho}\right\}
\end{aligned}
$$

with

$$
\begin{aligned}
\mathrm{G}_{2}^{\prime}= & t\left(\hat{F}_{00}^{51}-\frac{\hat{f}_{00}^{51}}{2}\right)+(1-\eta)\left(\hat{F}_{10}^{51}-\frac{\hat{f}_{10}^{51}}{2 t}\right) \\
& \left.+(1-\chi)\left(\hat{F}_{01}^{51}-\frac{\hat{f}_{01}^{51}}{2 t}\right)\right], \\
\mathrm{G}_{3}^{\prime}= & t\left[\left(\hat{F}_{10}^{51}-\frac{\hat{f}_{10}^{51}}{2 t}\right)+(1-\eta)\left(\hat{F}_{20}^{51}-\frac{\hat{f}_{20}^{51}}{2 t^{2}}\right)\right. \\
& \left.+(1-\chi)\left(\hat{F}_{11}^{51}-\frac{\hat{f}_{11}^{51}}{2 t^{2}}\right)\right], \\
\mathrm{G}_{4}^{\prime}= & t\left(\hat{F}_{01}^{51}-\frac{\hat{f}_{01}^{51}}{2 t}\right)+(1-\eta)\left(\hat{F}_{11}^{51}-\frac{\hat{f}_{11}^{51}}{2 t^{2}}\right) \\
& \left.+(1-\chi)\left(\hat{F}_{02}^{51}-\frac{\hat{f}_{02}^{51}}{2 t^{2}}\right)\right] .
\end{aligned}
$$

The meson momentum $k$ and the momentum transfer $q$ are connected to similar structures, but the functions $\mathrm{G}_{i}^{\prime}$ related to $k$ are far more complicated than $\mathrm{G}_{i}$. However, despite that fact the expressions within the parentheses in Eqs. (5.18)(5.20) could be written in the same manner as the expressions (5.11)-(5.14) showing potential WW-like expressions for the twist-2 distributions $\hat{f}_{n_{1} n_{2}}^{5 a}(t ; \eta, \chi)$.

For the symmetric part $T_{\{\mu \nu\}}^{\mathrm{tw} 2}$ of the Compton amplitude we perform the same calculational steps as in the antisymmetric part: namely, separation of $\mathcal{P}_{\rho} \mathcal{K}^{a \rho}$ contractions (this also includes the trace terms), insertion of $\mathcal{P}=P_{+} t+\pi z_{-}$ $+\tilde{\pi} z_{k}$ into the Compton amplitude (4.10), definition of the structure functions $\hat{F}_{n_{1} n_{2}}^{a}$ using Eq. (5.5), partial integration with respect to $t$ by formula (5.9), and projection onto the collinear part. After partial integration the symmetric part of the Compton amplitude has the following form:

$$
\begin{aligned}
T_{\{\mu \nu\}}^{\mathrm{tw} 2} \approx & -2 \int_{-1}^{1} d t \frac{1}{\xi+t-i \epsilon} \frac{1}{q P_{+}}\left\{g_{\mu \nu} q_{\rho} \hat{f}_{00}^{a}-\left(g_{\mu \rho} q_{\nu}+g_{\nu \rho} q_{\mu}\right) \hat{F}_{00}^{a}+\left(g_{\mu \rho} P_{+\nu}+g_{\nu \rho} P_{+\mu}\right) t \hat{F}_{00}^{a}\right. \\
& +\left(g_{\mu \rho} \pi_{\nu}+g_{\nu \rho} \pi_{\mu}\right) t \hat{F}_{10}^{a}+\left(g_{\mu \rho} \tilde{\pi}_{\nu}+g_{\nu \rho} \tilde{\pi}_{\mu}\right) t \hat{F}_{01}^{a}+\frac{q_{\rho}}{q P_{+}}\left[\left(q_{\mu} P_{+\nu}+q_{\nu} P_{+\mu}\right)\left(\hat{F}_{00}^{a}-\hat{f}_{00}^{a}\right)-P_{+\mu} P_{+\nu}\left(2 t \hat{F}_{00}^{a}-t \hat{f}_{00}^{a}\right)\right. \\
& +\left(q_{\mu} \pi_{\nu}+q_{\nu} \pi_{\mu}\right)\left(\hat{F}_{10}^{a}-\hat{f}_{10}^{a}\right)-\left(P_{+\mu} \pi_{\nu}+P_{+\nu} \pi_{\mu}\right)\left(t \hat{F}_{10}^{a}-t \hat{f}_{10}^{a}\right)+\left(q_{\mu} \tilde{\pi}_{\nu}+q_{\nu} \tilde{\pi}_{\mu}\right)\left(\hat{F}_{01}^{a}-\hat{f}_{01}^{a}\right)-\left(P_{+\mu} \tilde{\pi}_{\nu}+P_{+\nu} \tilde{\pi}_{\mu}\right) \\
& \left.\left.\times\left(t \hat{F}_{01}^{a}-t \hat{f}_{01}^{a}\right)-\pi_{\mu} \pi_{\nu}\left(2 t \hat{F}_{20}^{a}-t \hat{f}_{20}^{a}\right)-\tilde{\pi}_{\mu} \tilde{\pi}_{\nu}\left(2 t \hat{F}_{02}^{a}-t \hat{f}_{02}^{a}\right)-\left(\pi_{\mu} \tilde{\pi}_{\nu}+\pi_{\nu} \tilde{\pi}_{\mu}\right)\left(2 t \hat{F}_{11}^{a}-t \hat{f}_{11}^{a}\right)\right]\right\} \mathcal{K}^{a \rho} .
\end{aligned}
$$

The collinear part of $T_{\{\mu \nu\}}^{\mathrm{tw} 2}$, which contains only functions $\hat{F}_{00}^{a}$ and $\hat{f}_{00}^{a}$, can be written as 


$$
\begin{aligned}
T_{\{\mu \nu\}}^{\mathrm{tw} 2}= & -2 \int_{-1}^{1} d t \frac{1}{\xi+t-i \epsilon} \frac{1}{q P_{+}}\left\{q_{\rho}\left(g_{\mu \nu}-\frac{q_{\mu} P_{+\nu}+q_{\nu} P_{+\mu}}{q P_{+}}\right) \hat{f}_{00}^{a}(t ; \eta, \chi)+\frac{q_{\rho}}{2 q P_{+}} P_{+\mu} P_{+\nu} 2 t \hat{f}_{00}^{a}(t ; \eta, \chi)\right. \\
& -\left[q_{\mu}\left(g_{\nu \rho}-P_{+\nu} \frac{q_{\rho}}{q P_{+}}\right)+q_{\nu}\left(g_{\mu \rho}-P_{+\mu} \frac{q_{\rho}}{q P_{+}}\right)\right] \hat{F}_{00}^{a}(t ; \eta, \chi) \\
& \left.+\left[P_{+\mu}\left(g_{\nu \rho}-P_{+\nu} \frac{q_{\rho}}{q P_{+}}\right)+P_{+\nu}\left(g_{\mu \rho}-P_{+\mu} \frac{q_{\rho}}{q P_{+}}\right)\right] t \hat{F}_{00}^{a}(t ; \eta, \chi)\right\} \mathcal{K}^{a \rho} .
\end{aligned}
$$

Looking at the tensor structure of Eq. (5.22), the functions

$$
\begin{aligned}
& \mathrm{F}_{1}^{a}(t ; \eta, \chi)=\hat{f}_{00}^{a}(t ; \eta, \chi), \\
& \mathrm{F}_{2}^{a}(t ; \eta, \chi)=2 t \cdot \hat{f}_{00}^{a}(t ; \eta, \chi),
\end{aligned}
$$

appear as natural structure functions and obey a CallanGross-like relation [32]:

$$
\mathrm{F}_{2}^{a}(t ; \eta, \chi)=2 t \cdot \mathrm{F}_{1}^{a}(t ; \eta, \chi)
$$

Similar to Ref. [7] one observes that the remainder contributions in Eq. (5.22) are suppressed for large values of $\nu$, a property of the off-collinear terms. One may see this, contracting the structures $\mathcal{K}^{a \rho}$ with the respective tensors in front. As well known from forward scattering, the CallanGross relation receives corrections both from higher orders in the coupling constant and due to mass effects, see, e.g., Ref. [33], and therefore as well in the nonforward case. On the other hand, the Wandzura-Wilczek relation for geometric twist-2 turns out to be rigidly stable, see, e.g., Ref. [27].

Including also $\mathcal{P}_{\rho} \mathcal{K}^{a \rho}$ contractions in the calculation will result in an additional tensor structure analogous to Eq. (5.17) with $q_{\rho}$ replaced by $k_{\rho}$ in Eq. (5.22). The corresponding structure functions will not be given in explicit form.

\section{EVOLUTION EQUATIONS FOR THE DISTRIBUTION AMPLITUDES}

The scaling violations of the operator matrix elements and distribution amplitudes of the process considered are described by the renormalization group equations governing the ultraviolet behavior of the light-cone operators. The corresponding equations for the distribution amplitudes $f_{a}\left(z_{+}, z_{-}, z_{k}\right)$ are called evolution equations, which we are going to discuss in $x$ and $z$ space. Since the flavor content of the operators (2.7) and (2.8) has been suppressed in the preceding sections, we treat only the flavor nonsinglet evolution equations as an example. The singlet evolution equations are of quite similar structure (see, e.g., Ref. [6]; for earlier work see Refs. [34,35]).

The nonsinglet renormalization group equation for the twist- 2 vector operator reads

$$
\begin{aligned}
\mu^{2} \frac{d}{d \mu^{2}} O_{\beta}^{\mathrm{tw} 2}\left(\kappa_{1} \tilde{x}, \kappa_{2} \tilde{x} ; \mu^{2}\right)= & \int_{\mathbb{R}^{2}} D^{2} \kappa^{\prime} \gamma\left(\kappa_{1}, \kappa_{2} ; \kappa_{1}^{\prime}, \kappa_{2}^{\prime}\right) \\
& \times O_{\beta}^{\mathrm{tw} 2}\left(\kappa_{1}^{\prime} \tilde{x}, \kappa_{2}^{\prime} \tilde{x} ; \mu^{2}\right) .
\end{aligned}
$$

By contraction with $\tilde{x}^{\beta}$ it is obvious that the scalar twist-2 operator $O^{\text {tw } 2}=\tilde{x}^{\beta} O_{\beta}^{\text {tw } 2}$ obeys exactly the same renormalization group equation because multiplication with $\tilde{x}^{\beta}$ commutes with the differentiation on the left and with the integration on the right-hand side. This gives the renormalization group equation for the scalar operator which on the light cone already is of twist-2:

$$
\begin{aligned}
\mu^{2} \frac{d}{d \mu^{2}} O\left(\kappa_{1} \tilde{x}, \kappa_{2} \tilde{x} ; \mu^{2}\right)= & \int_{\mathrm{R}^{2}} D^{2} \kappa^{\prime} \gamma\left(\kappa_{1}, \kappa_{2} ; \kappa_{1}^{\prime}, \kappa_{2}^{\prime}\right) \\
& \times O\left(\kappa_{1}^{\prime} \tilde{x}, \kappa_{2}^{\prime} \tilde{x} ; \mu^{2}\right)
\end{aligned}
$$

In the last equations the integration measure

$$
D^{2} \kappa^{\prime} \equiv d \kappa_{1}^{\prime} d \kappa_{2}^{\prime} \theta\left(\kappa_{1}-\kappa_{1}^{\prime}\right) \theta\left(\kappa_{1}^{\prime}-\kappa_{2}\right) \theta\left(\kappa_{1}-\kappa_{2}^{\prime}\right) \theta\left(\kappa_{2}^{\prime}-\kappa_{2}\right)
$$

has been introduced. In Refs. [6,34] it is shown that the nonlocal anomalous dimension matrix $\gamma$ is invariant under translations and scale transformations,

$$
\begin{aligned}
\gamma\left(\kappa_{1}, \kappa_{2} ; \kappa_{1}^{\prime}, \kappa_{2}^{\prime}\right) & =\gamma\left(\kappa_{1}-\kappa_{0}, \kappa_{2}-\kappa_{0} ; \kappa_{1}^{\prime}-\kappa_{0}, \kappa_{2}^{\prime}-\kappa_{0}\right) \\
& =\lambda^{2} \gamma\left(\lambda \kappa_{1}, \lambda \kappa_{2} ; \lambda \kappa_{1}^{\prime}, \lambda \kappa_{2}^{\prime}\right)
\end{aligned}
$$

which reduces the number of independent variables of $\gamma$ by two. By first changing the variables from $\kappa_{1,2}$ to $\kappa_{ \pm}$, followed by a translation by $\kappa_{+}$and a scaling by $\kappa^{-1}$, one derives the following form of the evolution kernel $\gamma$ :

$$
\begin{aligned}
\gamma\left(\kappa_{1}, \kappa_{2} ; \kappa_{1}^{\prime}, \kappa_{2}^{\prime}\right) & =\tilde{\gamma}\left(\kappa_{+}, \kappa ; \kappa_{+}^{\prime}, \kappa^{\prime}\right) \\
& =\tilde{\gamma}\left(0, \kappa ; \kappa_{+}^{\prime}-\kappa_{+}, \kappa^{\prime}\right) \\
& =\frac{1}{\kappa^{2}} \tilde{\gamma}\left(0,1 ; \frac{\kappa_{+}^{\prime}-\kappa_{+}}{\kappa}, \frac{\kappa^{\prime}}{\kappa}\right) \\
& \equiv \frac{1}{\kappa^{2}} \tilde{K}\left(w_{1}, w_{2}\right)
\end{aligned}
$$

with 


$$
w_{1}=\frac{\kappa_{+}^{\prime}-\kappa_{+}}{\kappa} \text { and } w_{2}=\frac{\kappa^{\prime}}{\kappa}
$$

The variables $\kappa_{i}^{\prime}$ and $w_{i}$ are connected by the following transformation:

$$
\left(\begin{array}{l}
\kappa_{1}^{\prime} \\
\kappa_{2}^{\prime}
\end{array}\right)=\left(\begin{array}{cc}
\kappa & -\kappa \\
\kappa & \kappa
\end{array}\right)\left(\begin{array}{l}
w_{1} \\
w_{2}
\end{array}\right)+\left(\begin{array}{c}
\kappa_{+} \\
\kappa_{+}
\end{array}\right) .
$$

It is therefore more natural to use $w_{1}$ and $w_{2}$ as integration variables in the renormalization group equation (6.2) instead of $\kappa_{1}^{\prime}$ and $\kappa_{2}^{\prime}$. The integration measures are related by

$$
D^{2} \kappa^{\prime}=\kappa^{2} D^{2} w
$$

where $D^{2} \kappa^{\prime}$ and $D^{2} w$ include the suitable $\theta$ functions realizing the integration ranges of $\kappa_{i}^{\prime}(6.3)$ and $w_{i}$

$$
\begin{aligned}
D^{2} w \equiv & \frac{1}{2} d w_{1} d w_{2} \theta\left(1+w_{1}-w_{2}\right) \theta\left(1-w_{1}+w_{2}\right) \\
& \times \theta\left(1+w_{1}+w_{2}\right) \theta\left(1-w_{1}-w_{2}\right) .
\end{aligned}
$$

The measure $D^{2} w$ can be divided into two parts, because $\widetilde{K}\left(w_{1}, w_{2}\right)$ under the exchange $w_{1} \leftrightarrow-w_{1}, w_{2} \leftrightarrow-w_{2}$ obeys the following relations, cf. Ref. [6]:

$$
\widetilde{K}\left(w_{1}, w_{2}\right)=\widetilde{K}\left(-w_{1}, w_{2}\right)=-\widetilde{K}\left(w_{1},-w_{2}\right) .
$$

Setting Eqs. (6.5) and (6.8) into the renormalization group equation for the scalar operator results in

$$
\begin{aligned}
\mu^{2} \frac{d}{d \mu^{2}} O\left(\kappa_{1} \tilde{x}, \kappa_{2} \tilde{x} ; \mu^{2}\right)= & \int_{\mathbb{R}^{2}} D^{2} w \widetilde{K}\left(w_{1}, w_{2}\right) \\
& \times O\left(\kappa_{1}^{\prime} \tilde{x}, \kappa_{2}^{\prime} \tilde{x} ; \mu^{2}\right) .
\end{aligned}
$$

For the explicit structure of $\widetilde{K}\left(w_{1}, w_{2}\right)$ see Refs. [6,34,35]. Equation (6.10) will now be considered for the matrix elements of the scalar operator which are, according Eq. (3.20), given by

$$
\begin{aligned}
& \left\langle p_{2}, k\left|O\left(\kappa_{1} \tilde{x}, \kappa_{2} \tilde{x}, \mu^{2}\right)\right| p_{1}\right\rangle \\
& \quad=e^{i \kappa_{+} \tilde{x} P_{-}} \mathcal{K}^{a}\left(\tilde{x}, p_{2}, k, p_{1}\right) \widetilde{f}_{a}\left(\kappa \tilde{x} P_{+}, \kappa \tilde{x} P_{-}, \kappa \tilde{x} k, \mu^{2}\right) .
\end{aligned}
$$

From this one obtains directly the evolution equation for the distribution amplitudes $\widetilde{f}_{a}$ in $x$ space:

$$
\begin{aligned}
& \mu^{2} \frac{d}{d \mu^{2}} \tilde{f}_{a}\left(\kappa \tilde{x} P_{+}, \kappa \tilde{x} P_{-}, \kappa \tilde{x} k ; \mu^{2}\right) \\
& =\int_{\mathbb{R}^{2}} D^{2} w \widetilde{K}\left(w_{1}, w_{2}\right) e^{i w_{1} \kappa \tilde{x} P_{-}} \\
& \quad \times \widetilde{f}_{a}\left(w_{2} \kappa \tilde{x} P_{+}, w_{2} \kappa \tilde{x} P_{-}, w_{2} \kappa \tilde{x} k ; \mu^{2}\right) .
\end{aligned}
$$

Because we are interested in evolution equations in $z$ space, we perform a Fourier transformation of Eq. (6.12). The physically relevant transforms of $\widetilde{f}_{a}$ are given by

$$
\begin{aligned}
f_{a}\left(z_{+}, z_{-}, z_{k} ; \mu^{2}\right)= & \int_{\mathbb{R}} \frac{d\left(\kappa \tilde{x} P_{+}\right)}{2 \pi} \int_{\mathbb{R}} \frac{d\left(\kappa \tilde{x} P_{-}\right)}{2 \pi} \int_{\mathbb{R}} \frac{d(\kappa \tilde{x} k)}{2 \pi} \\
& \times e^{i \kappa \tilde{x}\left(P_{+} z_{+}+P_{-} z_{-}+k z_{k}\right)} \\
& \times \widetilde{f}_{a}\left(\kappa \tilde{x} P_{+}, \kappa \tilde{x} P_{-}, \kappa \tilde{x} k ; \mu^{2}\right) .
\end{aligned}
$$

Carrying out these transformations one arrives at the following result:

$$
\begin{aligned}
\mu^{2} \frac{d}{d \mu^{2}} f_{a}\left(z_{+}, z_{-}, z_{k}, \mu^{2}\right)= & \int_{\mathbb{R}} \frac{d\left(\kappa \tilde{x} P_{+}\right)}{2 \pi} \int_{\mathbb{R}} \frac{d\left(\kappa \tilde{x} P_{-}\right)}{2 \pi} \int_{\mathbb{R}} \frac{d(\kappa \widetilde{x} k)}{2 \pi} e^{i \kappa \widetilde{x}\left(P_{+} z_{+}+P_{-} z_{-}+k z_{k}\right)} \int_{\mathbb{R}^{2}} D^{2} w \widetilde{K}\left(w_{1}, w_{2}\right) \\
& \times \int_{\mathbb{R}^{3}} D^{3} z^{\prime} f_{a}\left(z_{+}^{\prime}, z_{-}^{\prime}, z_{k}^{\prime} ; \mu^{2}\right) e^{-i w_{2} \kappa \widetilde{x}\left(P_{+} z_{+}^{\prime}+P_{-} z_{-}^{\prime}+k z_{k}^{\prime}\right)} e^{i w_{1} \kappa \tilde{x} P_{-}} \\
= & \int_{\mathbb{R}^{2}} D^{2} w \widetilde{K}\left(w_{1}, w_{2}\right) \int_{\mathbb{R}^{3}} D^{3} z^{\prime} f_{a}\left(z_{+}^{\prime}, z_{-}^{\prime}, z_{k}^{\prime} ; \mu^{2}\right) \delta\left(z_{+}-w_{2} z_{+}^{\prime}\right) \delta\left(z_{-}-w_{2} z_{-}^{\prime}+w_{1}\right) \delta\left(z_{k}-w_{2} z_{k}^{\prime}\right) \\
= & \int_{\mathbb{R}^{2}} D^{2} z^{\prime} \frac{1}{\left|z_{+}\right|} \widetilde{K}\left(z_{-}^{\prime} \frac{z_{+}}{z_{+}^{\prime}}-z_{-}, \frac{z_{+}}{z_{+}^{\prime}}\right) f_{a}\left(z_{+}^{\prime}, z_{-}^{\prime}, z_{k} \frac{z_{+}^{\prime}}{z_{+}} ; \mu^{2}\right)
\end{aligned}
$$


with $D^{3} z^{\prime}$ given by Eq. (3.23) and $D^{2} z^{\prime}$ defined by

$$
\begin{aligned}
D^{2} z^{\prime}= & 2 d z_{+}^{\prime} d z_{-}^{\prime} \theta\left(1-z_{+}^{\prime}+z_{-}^{\prime}\right) \theta\left(1+z_{+}^{\prime}-z_{-}^{\prime}\right) \\
& \times \theta\left(1-z_{+}^{\prime}-z_{-}^{\prime}\right) \theta\left(1+z_{+}^{\prime}+z_{-}^{\prime}\right) .
\end{aligned}
$$

We introduce the evolution kernel

$$
\Gamma\left(z_{+}, z_{-}, z_{+}^{\prime}, z_{-}^{\prime}\right) \equiv \frac{1}{\left|z_{+}\right|} \widetilde{K}\left(z_{-}^{\prime} \frac{z_{+}}{z_{+}^{\prime}}-z_{-}, \frac{z_{+}}{z_{+}^{\prime}}\right),
$$

which leads to the this evolution equation

$$
\begin{aligned}
\mu^{2} \frac{d}{d \mu^{2}} f_{a}\left(z_{+}, z_{-}, z_{k} ; \mu^{2}\right)= & \int_{\mathbb{R}^{2}} D^{2} z^{\prime} \Gamma\left(z_{+}, z_{-}, z_{+}^{\prime}, z_{-}^{\prime}\right) \\
& \times f_{a}\left(z_{+}^{\prime}, z_{-}^{\prime}, z_{k} \frac{z_{+}^{\prime}}{z_{+}} ; \mu^{2}\right) .
\end{aligned}
$$

Let us point to the remarkable fact that the variable $z_{k}$ connected to the meson momentum $k$ only appears as a parameter in $f_{a}$ and is not contained in the evolution kernel $\Gamma$. The same observation has been made in Ref. [15] recently in the case of diffractive scattering, where the parameters $\eta$ or $x_{P}$ behave in the same way. Insofar some of the scaling variables of a problem, in the present case the variables $\chi_{i}$, play another role than others, as here $\xi$ and $\eta$, which interfere with the evolution.

This evolution equation is a fundamental equation because it describes the evolution of the triple-valued distribution amplitudes $f_{a}\left(z_{+}, z_{-}, z_{k}\right)$ in $z$ space. These amplitudes are the basic objects for the construction of the structure functions $F_{a}, F_{a}^{5}$, and $F_{a}^{\mathrm{tr}}$ in Eqs. (3.29) and (3.30). They are also used in the definition of the single-valued functions $\hat{F}_{n_{1} n_{2}}^{a}(t ; \eta, \chi)$ in Eq. (5.5). The scaling violations of these functions are obtained solving Eq. (6.16) and inserting the functions $f_{a}$ into Eqs. (3.29) and (3.30).

It is also possible to obtain another evolution equation for $\hat{f}_{00}^{a}(t ; \eta, \chi)$ in the variable $t$, which is compatible with the former equation. This single-variable evolution equation governs the evolution of the structure functions contained in the collinear part of the Compton amplitude.

To begin with, we first show that the distribution amplitude $\hat{f}_{00}^{a}(t ; \eta, \chi)$ given by

$$
\hat{f}_{00}^{a}(t ; \eta, \chi)=\int d z_{-} \int d z_{k} \hat{f}^{a}\left(t-\eta z_{-}-\chi z_{k}, z_{-}, z_{k}\right)
$$

has another representation obtained as

$$
\begin{aligned}
\mathcal{K}^{a}(\mathbf{p}) \hat{f}_{00}^{a}(t ; \eta, \chi) \\
=\int \frac{d\left(\kappa \tilde{x} P_{+}\right)}{2 \pi} e^{i \kappa \tilde{x} P_{+}} \\
\quad \times\left.\left\langle p_{2}, k|O(-\kappa \tilde{x}, \kappa \tilde{x})| p_{1}\right\rangle\right|_{\tilde{x} P_{-}=\eta \tilde{x} P_{+} ; \tilde{x} k=\chi \tilde{x} P_{+}} .
\end{aligned}
$$

The constraints

$$
\begin{gathered}
\tilde{x} P_{-}=\eta \tilde{x} P_{+}, \\
\tilde{x} k=\chi \tilde{x} P_{+},
\end{gathered}
$$

appearing in the former equation are the scaling relations (1.7) in $x$ space. Using the representation (3.25) under these constraints leads to the result (6.17).

To derive the single-variable evolution equation we form matrix elements of Eq. (6.10),

$$
\begin{aligned}
\left.\mu^{2} \frac{d}{d \mu^{2}}\left\langle p_{2}, k|O(-\kappa \tilde{x}, \kappa \tilde{x})| p_{1}\right\rangle\right|_{\tilde{x} P_{-}=\eta \tilde{x} P_{+} ; \tilde{x} k=\chi \tilde{x} P_{+}} \\
=\int_{\mathbb{R}^{2}} D^{2} w \widetilde{K}\left(w_{1}, w_{2}\right) e^{i w_{1} \kappa \tilde{x} P_{-}} \\
\quad \times\left.\left\langle p_{2}, k\left|O\left(-w_{2} \kappa \tilde{x}, w_{2} \kappa \tilde{x}\right)\right| p_{1}\right\rangle\right|_{\tilde{x} P_{-}=\eta \tilde{x} P_{+} ; \tilde{x} k=\chi \tilde{x} P_{+}},
\end{aligned}
$$

and perform the Fourier transformation in the variable $\kappa \tilde{x} P_{+}$ according to Eq. (6.18). The direct calculation leads to

$$
\mu^{2} \frac{d}{d \mu^{2}} \hat{f}_{00}^{a}\left(t ; \eta, \chi, \mu^{2}\right)=\int_{-1}^{1} d t^{\prime} \gamma\left(t, t^{\prime} ; \eta\right) \hat{f}_{00}^{a}\left(t^{\prime} ; \eta, \chi, \mu^{2}\right),
$$

with the evolution kernel

$$
\gamma\left(t, t^{\prime} ; \eta\right)=\int d w_{2} \frac{1}{|\eta|} \widetilde{K}\left(\frac{w_{2} t^{\prime}-t}{\eta}, w_{2}\right) .
$$

Like the evolution kernel $\Gamma\left(z_{+}, z_{-} ; z_{+}^{\prime}, z_{-}^{\prime}\right)$, also $\gamma\left(t, t^{\prime} ; \eta\right)$ does not depend on any $k$-dependent variables like $\chi$ or $z_{k}$ being related to the meson momentum.

\section{GENERALIZATION TO AN ARBITRARY NUMBER OF OUTGOING MESONS}

In this section we summarize the generic properties of the results obtained in the preceding sections and extend it to an arbitrary number of outgoing mesons. Generally, one may state that all the above results remain valid under slight modifications if two or more outgoing scalar mesons are present in the process,

$$
\gamma_{1}^{*}\left(q_{1}\right)+H\left(p_{1}\right) \rightarrow \gamma_{2}^{*}\left(q_{2}\right)+H\left(p_{2}\right)+M\left(k_{1}\right)+\cdots+M\left(k_{n}\right),
$$




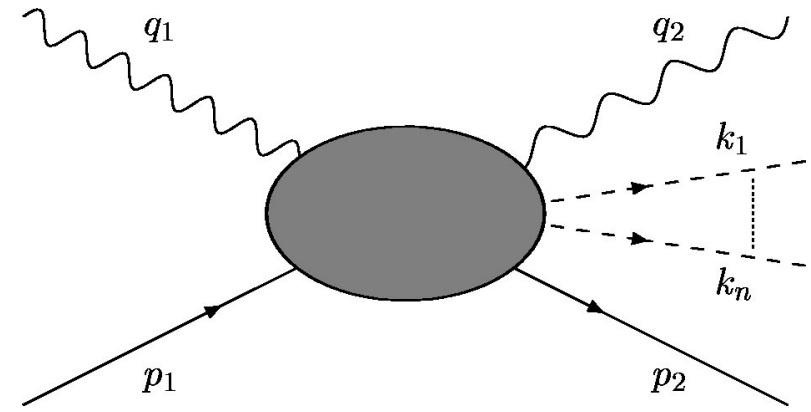

FIG. 1. Process with $n$ outgoing mesons.

as shown in Fig. 1. To fix the kinematic domain of this process, all meson momenta $k_{i}$ are connected to different scaling variables $\chi_{i}$ defined by

$$
\chi_{i}=\frac{k_{i}}{q P_{+}}
$$

where $P_{+}$and $P_{-}$are obviously given by

$$
P_{ \pm}=\left(p_{2}+\sum_{i=1}^{n} k_{i}\right) \pm p_{1}
$$

and $\xi$ and $\eta$ are introduced as in Eq. (1.7). In order to compute the twist-2 part of the Compton amplitude

$$
\begin{aligned}
T_{\mu \nu}\left(p_{2}, k_{1}, \ldots, k_{n}, p_{1}, q\right) \\
=i \int d^{4} x e^{i q x}\left\langle p_{2}, S_{2} ; k_{1}, \ldots, k_{n}\right| \\
\quad \times R T\left[J_{\mu}\left(\frac{x}{2}\right) J_{\nu}\left(-\frac{x}{2}\right) \mathcal{S}\right]\left|p_{1}, S_{1}\right\rangle
\end{aligned}
$$

for the general process (7.1) one applies the same approximations to the operator $\hat{T}_{\mu \nu}$ as in Secs.. II and III. Thus one uses the approximation (2.12) and applies the twist-2 projection (3.6).

The technique used in Sec. III to construct the matrix elements $\left\langle p_{2}, k\left|O^{(5)}\right| p_{1}\right\rangle$ can be carried out for an arbitrary number $n$ of scalar mesons where the additional meson momenta $\left\{k_{2}, \ldots, k_{n}\right\}$ enlarge the set of kinematic factors $\mathcal{K}^{a}(\tilde{x}, \mathbf{p})$. However, these factors are easy to guess and their number is given by

$$
N_{\text {scalar }}=\left\{\begin{array}{l}
2^{n+1}+\left(\begin{array}{c}
n+3 \\
4
\end{array}\right) \text { for } n \geqslant 1 \\
2 \text { for } n=0,
\end{array}\right.
$$

for the scalar matrix element. This formula also reproduces the number of kinematic factors in the ordinary nonforward case: the Dirac and Pauli structures.

Having all kinematic factors $\mathcal{K}^{a}$ at hand, one introduces the related structure functions $\widetilde{f}_{a}$ and writes down the following decomposition of the scalar matrix element

$$
\begin{aligned}
\left\langle p_{2}, k_{1}, \ldots, k_{k}|O(-\kappa \tilde{x}, \kappa \tilde{x})| p_{1}\right\rangle \\
=\sum_{a=1}^{N_{\text {scalar }}} \mathcal{K}_{a}\left(\tilde{x}, p_{2}, k_{1}, \ldots, k_{n}, p_{1}\right) \\
\quad \times \tilde{f}_{a}\left(\kappa \tilde{x} P_{+}, \kappa \tilde{x} P_{-}, \kappa \tilde{x} k_{1}, \ldots, \kappa \tilde{x} k_{n}, \mathbf{p}_{i} \mathbf{p}_{j} ; \mu^{2}\right) .
\end{aligned}
$$

With this representation one goes through the same steps of the calculation as in the preceding sections: namely,

Fourier transformation of $\widetilde{f}_{a}$, application of the twist-2 projector, and computation of the Compton amplitude.

The result is again of the form (4.5) with a larger $z$ space and $\mathcal{P}$ given by

$$
\mathcal{P}=P_{+} z_{+}+P_{-} z_{-}+\sum_{i=1}^{n} \chi_{i} z_{k_{i}}
$$

In this sense, the form (4.5) of the Compton amplitude is a generic result holding for a large class of processes. The more explicit form (4.10) is generalized by replacing $\chi z_{k}$ by $\sum \chi_{i} z_{k_{i}}$

It is even possible to interpret the Wandzura-Wilczek and Callan-Gross relations obtained in Sec. V as generic properties of the collinear parts of these processes. Making the substitution

$$
\mathcal{P}=P_{+} t+\pi z_{-}+\sum_{i=1}^{n} \tilde{\pi}_{i} z_{k_{i}} \quad \text { with } \quad \tilde{\pi}_{i}=k_{i}-\chi_{i} P_{+},
$$

and projecting onto the collinear part one finds again the relations

$$
\begin{aligned}
\mathrm{G}_{2}^{a}\left(t ; \eta, \chi_{1}, \ldots, \chi_{n}\right)= & -\mathrm{G}_{1}^{a}\left(t ; \eta, \chi_{1}, \ldots, \chi_{n}\right) \\
& +\int_{t}^{\operatorname{sign}(t)} \frac{d \lambda}{\lambda} \mathrm{G}_{1}^{a}\left(\lambda ; \eta, \chi_{1}, \ldots, \chi_{n}\right),
\end{aligned}
$$

$$
\mathrm{F}_{2}^{a}\left(t ; \eta, \chi_{1}, \ldots, \chi_{n}\right)=2 t \cdot \mathrm{F}_{1}^{a}\left(t ; \eta, \chi_{1}, \ldots, \chi_{n}\right)
$$

Looking at the derivation of the evolution equation in Sec. VI, it is not difficult to find the appropriate generalization to an arbitrary number of mesons $\left[\mathbf{k}=\left(k_{1}, \ldots, k_{n}\right)\right]$ :

$$
\begin{aligned}
& \mu^{2} \frac{d}{d \mu^{2}} f_{a}\left(z_{+}, z_{-}, z_{\mathbf{k}} ; \mu^{2}\right) \\
& =\int_{\mathbb{R}^{2}} D^{2} z^{\prime} \frac{\left|z_{+}^{\prime}\right|^{n-1}}{\left|z_{+}\right|^{n}} \widetilde{K}\left(z_{-}^{\prime} \frac{z_{+}}{z_{+}^{\prime}}-z_{-}, \frac{z_{+}}{z_{+}^{\prime}}\right) \\
& \quad \times f_{a}\left(z_{+}^{\prime}, z_{-}^{\prime}, z_{\mathbf{k}} \frac{z_{+}^{\prime}}{z_{+}} ; \mu^{2}\right) .
\end{aligned}
$$

For $n=0$, this general evolution equation is reproducing the flavor nonsinglet part of the evolution equation given in Ref. [6] for the ordinary nonforward scattering. As in the case of 
one meson in Sec. VI the variables $z_{k_{i}}$ connected to the meson momenta only appear as parameters and do not contribute to the evolution kernel,

$$
\Gamma^{n}\left(z_{+}, z_{-}, z_{+}^{\prime}, z_{-}^{\prime}\right) \equiv \frac{\left|z_{+}^{\prime}\right|^{n-1}}{\left|z_{+}\right|^{n}} \widetilde{K}\left(z_{-}^{\prime} \frac{z_{+}}{z_{+}^{\prime}}-z_{-}, \frac{z_{+}}{z_{+}^{\prime}}\right) .
$$

Only the number of mesons is relevant for the structure of this kernel. The single-variable evolution equation is of the same form as in the preceding section. One only has to enlarge the number of scaling parameters $\chi_{i}$,

$$
\begin{aligned}
& \mu^{2} \frac{d}{d \mu^{2}} \hat{f}_{00}^{a}\left(t ; \eta, \chi_{1}, \ldots, \chi_{n}, \mu^{2}\right) \\
& \quad=\int_{-1}^{1} d t^{\prime} \gamma\left(t, t^{\prime} ; \eta\right) \hat{f}_{00}^{a}\left(t^{\prime} ; \eta, \chi_{1}, \ldots, \chi_{n}, \mu^{2}\right) .
\end{aligned}
$$

\section{CONCLUSIONS}

We studied the structure of the virtual Compton amplitude for deep-inelastic nonforward scattering $\gamma^{*}\left(q_{1}\right)+H\left(p_{1}\right)$ $\rightarrow \gamma^{*}\left(q_{2}\right)+H\left(p_{2}\right)+M(k)$ at the level of the twist-2 contributions in lowest order in QCD in the massless limit. In the extended Bjorken region, i.e., $\left\{\left(q P_{+}\right),-q^{2}\right\} \rightarrow \infty$ with $-q^{2} /\left(q P_{+}\right),\left(q P_{-}\right) /\left(q P_{+}\right)$and $\left(k P_{-}\right) /\left(q P_{+}\right)$kept fixed, the twist-2 contributions to the Compton amplitude were calculated using the non-local operator product expansion for general spin states. In this approximation the Compton amplitude consists of five kinematically independent parts which in the limit $k \rightarrow 0$ reduce to the well known Dirac- and Pauli-type amplitudes. A decomposition of the Compton amplitude was performed with respect to the helicity states of both (virtual) photons. In complete analogy the (electromagnetic) gauge invariance of the nonlocal light-cone expansion holds at the level of the $S$ matrix since the fact that the leptonic currents are conserved. Due to this, only those contributions in the Compton amplitude are projected out, which obey gauge invariance. Integral relations generalizing the Callan-Gross and Wandzura-Wilczek relations for unpolarized and polarized forward-scattering are derived by reduction to the collinear parts of the Compton amplitude and, thereby, reducing the triple-valued distribution amplitudes to one-valued ones [for $\left(q P_{-}\right) /\left(q P_{+}\right)$and $\left(k P_{-}\right) /\left(q P_{+}\right)$ fixed]. In this connection attention has been drawn to the difference between geometric and dynamic WW relations being related to different notions of twist. The evolution kernels of these distribution amplitudes are obtained from the (well-known) nonlocal anomalous dimensions of the (scalar) twist-2 light-ray operators $O^{\text {tw } 2}\left(\kappa_{1} \tilde{x}, \kappa_{2} \tilde{x}\right)$ and $O^{5 \text { tw } 2}\left(\kappa_{1} \tilde{x}, \kappa_{2} \tilde{x}\right)$; they are independent of the meson momentum $k$. These results show that deeply virtual Compton scattering off nucleons in the case of additional meson production behaves quite similar to the case where mesons are absent. Both the basic structural relations as well as the scal- ing violations are the same in both cases. However, the structure of the Compton amplitude is different in general, however, only with corrections of $\mathcal{O}(1 / \sqrt{\nu})$ or less.

\section{ACKNOWLEDGMENTS}

The authors are grateful to M. Lazar and M. Diehl for various useful discussions. In addition, J. Eilers gratefully acknowledges the Graduate College "Quantum field theory" at Center for Theoretical Studies of Leipzig University for financial support.

\section{APPENDIX A: PROJECTION OF THE TWIST-2 OPERATOR ONTO THE LIGHT CONE}

This appendix is devoted to the derivation of the results (3.32) and (3.33) from the off-cone twist-2 nonlocal quarkantiquark operators obtained in Ref. [18]. As has been shown there the nonlocal quark-antiquark operator of geometric twist-2 has the following structure $\left(\left(\bar{\psi} \gamma_{\mu} \psi\right)(q)\right.$ $\left.=\int d^{4} x \exp \{i(q x)\} \bar{\psi}(x) \gamma_{\mu} \psi(-x)\right)$ :

$$
\begin{aligned}
O_{\alpha}^{\mathrm{tw} 2}(x,-x)= & \int \frac{d^{4} q}{(2 \pi)^{4}}\left(\bar{\psi} \gamma_{\mu} \psi\right)(q)\left(2+q \partial_{q}\right) \int_{0}^{1} d \tau \\
& \times\left\{\left[\left(3+q \partial_{q}\right) \delta_{\alpha}^{\mu}-i \tau q^{\mu} x_{\alpha}\right] \mathcal{H}_{2}(q \mid \tau x)\right. \\
& +\left[( 3 + q \partial _ { q } ) \left(\left(4+q \partial_{q}\right) i t q_{\alpha} x^{\mu}\right.\right. \\
& \left.-\frac{1}{2}(i \tau)^{2}\left(q^{2} x^{\mu} x_{\alpha}+x^{2} q^{\mu} q_{\alpha}\right)\right) \\
& \left.\left.+\frac{1}{4}(i t)^{3} q^{\mu} q^{2} x_{\alpha} x^{2}\right] \mathcal{H}_{3}(q \mid \tau x)\right\} .
\end{aligned}
$$

Its $n$th moment is given by

$$
\begin{aligned}
O_{\alpha n}^{\mathrm{tw} 2}(x)= & \frac{1}{(n+1)^{2}} \int \frac{d^{4} q}{(2 \pi)^{4}}\left(\bar{\psi} \gamma_{\mu} \psi\right)(q) \\
& \times\left\{\delta_{\alpha}^{\mu} h_{n}^{2}(q \mid x)-q^{\mu} x_{\alpha} h_{n-1}^{2}(q \mid x)\right. \\
& +2 x^{\mu} q_{\alpha} h_{n-1}^{3}(q \mid x) \\
& -\left(x^{\mu} x_{\alpha} q^{2}+q^{\mu} q_{\alpha} x^{2}\right) h_{n-2}^{3}(q \mid x) \\
& \left.+\frac{1}{2} q^{\mu} x_{\alpha} x^{2} q^{2} h_{n-3}^{3}(q \mid x)\right\} .
\end{aligned}
$$

Here, for notational simplicity we used the following abbreviations:

$$
\begin{aligned}
\mathcal{H}_{\nu}(q \mid x)= & \sqrt{\pi}\left[\sqrt{(q x)^{2}-q^{2} x^{2}}\right]^{1 / 2-\nu} J_{\nu-1 / 2}\left(\frac{1}{2} \sqrt{(q x)^{2}-q^{2} x^{2}}\right) \\
& \times e^{i q x / 2}, \\
h_{n}^{\nu}(q \mid x)= & \left(\frac{1}{2} \sqrt{q^{2} x^{2}}\right)^{n} C_{n}^{\nu}\left(\frac{q x}{\sqrt{q^{2} x^{2}}}\right) .
\end{aligned}
$$


The relation between the nonlocal and the local operators, Eqs. (A1) and (A2), off the light cone is obtained by observing that the Bessel functions are generating functions of the Gegenbauer polynomials (see, e.g., Ref. [36], Eq. II.5.13.1.3):

$$
\begin{aligned}
\sum_{n=0}^{\infty} \frac{a^{n}}{(2 \nu)_{n}} C_{n}^{\nu}(z)= & \Gamma\left(\nu+\frac{1}{2}\right) \\
& \times\left(\frac{a}{2} \sqrt{1-z^{2}}\right)^{1 / 2-\nu} J_{\nu-1 / 2}\left(a \sqrt{1-z^{2}}\right) e^{z a}
\end{aligned}
$$

where

$$
\begin{aligned}
(2 \nu)_{n} & =2 \nu(2 \nu+1) \cdots(2 \nu+n-1) \\
& =\Gamma(n+2 \nu) / \Gamma(2 \nu)
\end{aligned}
$$

is the Pochhammer symbol.

The projection onto the light cone is obtained most easily by first considering the local operators. Because of the series expansion of the Gegenbauer polynomials (see, e.g., Ref. [36], Appendix II.11),

$$
C_{n}^{\nu}(z)=\frac{1}{(\nu-1) !} \sum_{k=0}^{[n / 2]} \frac{(-1)^{k}(n-k+\nu-1) !}{k !(n-2 k) !}(2 z)^{n-2 k},
$$

one observes that from the expression (A4) on the light cone, $x^{2}=0$, only the term with the highest power, i.e., for $k=0$, survives

$$
h_{n}^{\nu}(q \mid \tilde{x})=\frac{(n+\nu-1) !}{n !(\nu-1) !}(q \tilde{x})^{n} .
$$

Using these results in the expression (A2) we obtain

$$
\begin{aligned}
O_{\alpha n}^{\mathrm{tw} 2}(\tilde{x})= & \frac{1}{n+1} \int \frac{d^{4} q}{(2 \pi)^{4}} \\
& \times\left(\bar{\psi} \gamma_{\mu} \psi\right)(q)\left\{\delta_{\alpha}^{\mu}(q \tilde{x})^{n}+\tilde{x}^{\mu} q_{\alpha} n(q \tilde{x})^{n-1}\right\} \\
& -\frac{1}{(n+1)^{2}} \int \frac{d^{4} q}{(2 \pi)^{4}}\left(\bar{\psi} \gamma_{\mu} \psi\right)(q) \tilde{x}_{\alpha} \\
& \times\left\{q^{\mu} n(q \tilde{x})^{n-1}+\frac{1}{2} \tilde{x}^{\mu} q^{2} n(n-1)(q \tilde{x})^{n-2}\right\} .
\end{aligned}
$$

Now, using

$$
\frac{1}{n+1}=\int_{0}^{1} d \tau \tau^{n} \quad \text { and } \quad \frac{1}{(n+1)^{2}}=-\int_{0}^{1} d \tau \tau^{n} \ln \tau
$$

we are able to resume over $n$ according to

$$
O_{\alpha}^{\mathrm{tw} 2}(-\kappa \tilde{x}, \kappa \tilde{x})=\sum_{n=0}^{\infty} \frac{(-i \kappa)^{n}}{n !} O_{\alpha n}^{\mathrm{tw} 2}(\tilde{x}) .
$$

There are two options of doing this. In the first instance we may replace $i \kappa q_{\mu}$ in Eq. (A8) by the derivative $\widetilde{\partial}_{\mu}$ acting on the exponential $\exp \{i \kappa(q \tilde{x})\}$. This way one retains the expression (3.5) of the nonlocal twist-2 operator on the light cone from which the expression (3.32) has been derived. On the other hand, after taking matrix elements of Eq. (A1) and observing the definitions (3.29) and (3.30) of the distribution amplitudes $F_{a}(\mathbf{z})$ and $F_{a}^{\mathrm{tr}}(\mathbf{z})$, one obtains exactly the expression (3.32). Analogous results hold for the axial vector case (3.33).

The same result could have been obtained also using the Poisson integral for the Bessel functions (cf. Ref. [37], Eq. II.7.12.7),

$$
\Gamma\left(\nu+\frac{1}{2}\right) J_{\nu}(z)=\frac{1}{\sqrt{\pi}}\left(\frac{z}{2}\right)^{\nu} \int_{-1}^{1} d t\left(1-t^{2}\right)^{\nu-1 / 2} e^{i t z}
$$

for $\operatorname{Re} \nu>-\frac{1}{2}$, in order to express the functions (A3) on the light cone by

$$
\mathcal{H}_{\nu}(q \mid \tilde{x})=\frac{1}{\Gamma(\nu)} \int_{0}^{1} d \lambda[\lambda(1-\lambda)]^{\nu-1} e^{i \lambda(q \tilde{x})} .
$$

Then, after shifting the homogeneous derivations $q \partial_{q}$ in the expression (A1) to the right and interpreting it as $\lambda \partial_{\lambda}$ acting on the exponential, some partial integrations with respect to $\lambda$ can be performed which, finally, lead again to the expressions (3.5) and (3.32), respectively.

\section{APPENDIX B: HELICITY PROJECTIONS AND CURRENT CONSERVATION}

In this appendix we construct the helicity projections of the Compton amplitude generalizing the results of Ref. [7] to the present case. We start with the construction of the helicity basis of the two virtual photons $\gamma_{1}^{*}$ and $\gamma_{2}^{*}$. To simplify this construction, we choose the Breit frame, in which the relevant momenta read

$$
\begin{aligned}
P_{+} & =(\mu ; \overrightarrow{0}), \\
P_{-} & =\left(0 ; 0,0, P_{-3}\right), \\
k & =\left(k_{0} ; k_{1}, k_{2}, k_{3}\right), \\
q & =\left(q_{0} ; q_{1}, 0, q_{3}\right),
\end{aligned}
$$

where $\mu$ is introduced as a mass scale of the hadronic momentum $P_{+}$with $P_{+}^{2}=\mu^{2}$. To define the helicity basis we introduce the two reference vectors

$$
\begin{aligned}
& n_{0}=(1 ; 0,0,0), \\
& n_{2}=(0 ; 0,1,0) .
\end{aligned}
$$


The polarization vectors of the photons $\gamma_{1}^{*}$ and $\gamma_{2}^{*}$ are then given by

$$
\begin{aligned}
& \varepsilon_{0 \mu}^{1}=\frac{1}{N_{01}} q_{1 \mu}, \\
& \varepsilon_{0 \mu}^{2}=\frac{1}{N_{02}} q_{2 \mu}, \\
& \varepsilon_{1 \mu}^{1}=\varepsilon_{1 \mu}^{2}=n_{2 \mu}, \\
& \varepsilon_{2 \mu}^{1}=\frac{1}{N_{21}} \varepsilon_{\mu \alpha \beta \gamma} n_{0}^{\alpha} n_{2}^{\beta} q_{1}^{\gamma},
\end{aligned}
$$

$$
\varepsilon_{2 \mu}^{2}=\frac{1}{N_{22}} \varepsilon_{\mu \alpha \beta \gamma} n_{0}^{\alpha} n_{2}^{\beta} q_{2}^{\gamma}
$$

$$
\varepsilon_{3 \mu}^{1}=\frac{1}{N_{31}}\left(q_{1 \mu} q_{1} \cdot n_{0}-n_{0 \mu} q_{1} \cdot q_{1}\right)
$$

The normalization factors are given by

$$
\begin{aligned}
& N_{01}=\nu \sqrt{\left|\xi-\eta-\frac{P_{-}^{2}}{4 \nu}\right|}, \\
& N_{02}=\nu^{1 / 2} \sqrt{\left|\xi+\eta-\frac{P_{-}^{2}}{4 \nu}\right|}, \\
& N_{21}=\frac{\nu}{\mu} \sqrt{\mid 1+\frac{\mu^{2}}{\nu}(\xi-\eta)-\frac{\mu^{2} P_{-}^{2}}{4 \nu^{2} \mid},}, \\
& N_{22}=\frac{\nu}{\mu} \sqrt{\mid 1+\frac{\mu^{2}}{\nu}(\xi+\eta)-\frac{\mu^{2} P_{-}^{2}}{4 \nu^{2} \mid},} \\
& N_{31}=\frac{\nu^{3 / 2}}{\mu} \sqrt{\left|\xi-\eta+\frac{\mu^{2}}{\nu}(\xi-\eta)^{2}-P_{-}^{2}\left(\frac{1}{4 \nu}-\frac{\mu^{2}}{2 \nu^{2}}(\xi-\eta)+\frac{\mu^{2} P_{-}^{2}}{16 \nu^{3}}\right)\right|}, \\
& N_{32}=\frac{\nu^{3 / 2}}{\mu} \sqrt{\left|\xi+\eta+\frac{\mu^{2}}{\nu}(\xi+\eta)^{2}-P_{-}^{2}\left(\frac{1}{4 \nu}-\frac{\mu^{2}}{2 \nu^{2}}(\xi+\eta)+\frac{\mu^{2} P_{-}^{2}}{16 \nu^{3}}\right)\right|},
\end{aligned}
$$

which follows from the relations

$$
\begin{aligned}
& \left(q P_{+}\right)=\nu, \\
& \left(q P_{-}\right)=\eta \nu, \\
& (q q)=-\xi \nu, \\
& \left(q n_{0}\right)=\frac{\nu}{\mu}
\end{aligned}
$$

[see definitions (1.7) and note that $n_{0}=P_{+} / \mu$ in the Breit frame] and conservation of momentum

$$
q_{1}=q+\frac{P_{-}}{2}
$$

$$
q_{2}=q-\frac{P_{-}}{2} .
$$

The polarization vectors obey the following normalization condition:

$$
\varepsilon_{a \mu}^{i} \varepsilon_{b}^{i \mu}=s_{a} \delta_{a b},
$$

with $s_{a}=-1$ for $a=0,1,2$ and $s_{a}=1$ for $a=3$. We now use this helicity basis to compute all matrix elements

$$
\mathrm{T}_{k l} \equiv \varepsilon_{k}^{2 \mu} T_{\mu \nu}^{\mathrm{tw} 2} \varepsilon_{l}^{1 \nu} \quad \text { with } \quad k, l \in\{0,1,2,3\} .
$$

The result of the straightforward calculation is

$\mathrm{T}_{00}^{F} \approx \frac{2}{\nu} \frac{1}{\sqrt{\left|\xi^{2}-\eta^{2}\right|}} \int_{\mathbb{R}^{3}} D^{3} z F_{a}\left(z_{+}, z_{-}, z_{k}\right) q_{\rho} \mathcal{K}^{a \rho}$, 


$$
\begin{aligned}
& \mathrm{T}_{11}^{F} \approx 2 \int_{\mathrm{R}^{3}} D^{3} z\left(-\frac{1}{\mathcal{Q}^{2}+i \epsilon}+\frac{q \cdot \mathcal{P}}{\left(\mathcal{Q}^{2}+i \epsilon\right)^{2}}\right) F_{a} q_{\rho} \mathcal{K}^{a \rho}, \\
& \mathrm{T}_{22}^{F} \approx 2 \int_{\mathrm{R}^{3}} D^{3} z\left(-\frac{1}{\mathcal{Q}^{2}+i \epsilon}+\frac{q \cdot \mathcal{P}}{\left(\mathcal{Q}^{2}+i \epsilon\right)^{2}}\right) F_{a} q_{\rho} \mathcal{K}^{a \rho},
\end{aligned}
$$$$
\mathrm{T}_{33}^{F} \approx \frac{2}{\nu} \frac{1}{\sqrt{\left|\xi^{2}-\eta^{2}\right|} \sqrt{\left|1+\frac{M^{2}}{\nu} \xi+\frac{M^{4}}{\nu^{2}}\left(\xi^{2}-\eta^{2}\right)\right|}}
$$$$
\times \int_{\mathbb{R}^{3}} D^{3} z F_{a}\left(z_{+}, z_{-}, z_{k}\right) q_{\rho} \mathcal{K}^{a \rho},
$$$$
\mathrm{T}_{01}^{F}, \mathrm{~T}_{10}^{F}, \mathrm{~T}_{02}^{F}, \mathrm{~T}_{20}^{F}=O\left(\frac{1}{\sqrt{\nu}}\right) \text {, }
$$

$$
\begin{aligned}
\mathrm{T}_{03}^{F} \approx & \frac{2}{\nu} \frac{1}{\sqrt{\left|\xi^{2}-\eta^{2}\right|} \sqrt{\left|1+\frac{M^{2}}{\nu}(\xi-\eta)\right|}} \\
& \times \int_{\mathbb{R}^{3}} D^{3} z F_{a}\left(z_{+}, z_{-}, z_{k}\right) q_{\rho} \mathcal{K}^{a \rho},
\end{aligned}
$$

$$
\begin{array}{r}
\mathrm{T}_{30}^{F} \approx \frac{2}{\nu} \frac{1}{\sqrt{\left|\xi^{2}-\eta^{2}\right|} \sqrt{\left|1+\frac{M^{2}}{\nu}(\xi+\eta)\right|}} \\
\times \int_{\mathbb{R}^{3}} D^{3} z F_{a}\left(z_{+}, z_{-}, z_{k}\right) q_{\rho} \mathcal{K}^{a \rho}, \\
\mathrm{T}_{12}^{F}, \mathrm{~T}_{21}^{F}=O\left(\frac{1}{\nu}\right), \\
\mathrm{T}_{13}^{F}, \mathrm{~T}_{31}^{F}, \mathrm{~T}_{23}^{F}, \mathrm{~T}_{32}^{F}=O\left(\frac{1}{\sqrt{\nu}}\right)
\end{array}
$$

for the symmetric part. The helicity projections of the trace terms are all of order $1 / \nu$ and therefore not given in explicit form. The same calculation is carried out for the antisymmetric part:

$$
\begin{gathered}
\mathrm{T}_{01}^{F 5}, \mathrm{~T}_{10}^{F 5}, \mathrm{~T}_{02}^{F 5}, \mathrm{~T}_{20}^{F 5}=O\left(\frac{1}{\sqrt{\nu}}\right), \\
\mathrm{T}_{03}^{F 5}, \mathrm{~T}_{30}^{F 5}=O\left(\frac{1}{\nu}\right), \\
\mathrm{T}_{12}^{F 5} \approx 2 \int_{\mathbb{R}^{3}} D^{3} z\left(\frac{1}{\mathcal{Q}^{2}+i \epsilon}-\frac{q \mathcal{P}}{\left(\mathcal{Q}^{2}+i \epsilon\right)^{2}}\right) F_{a}^{5} q_{\rho} \mathcal{K}^{5 a \rho}, \\
\mathrm{T}_{21}^{F 5} \approx 2 \int_{\mathbb{R}^{3}}^{F 5}, \mathrm{~T}_{32}^{F 5}=O\left(\frac{1}{\sqrt{\nu}}\right), \\
\left.-\frac{1}{\mathcal{Q}^{2}+i \epsilon}+\frac{q \mathcal{P}}{\left(\mathcal{Q}^{2}+i \epsilon\right)^{2}}\right) F_{a}^{5} q_{\rho} \mathcal{K}^{5 a \rho} .
\end{gathered}
$$

Here, we have only kept terms contributing to the highest power in $\nu$, because all other terms vanish in the limit $\nu$ $\rightarrow \infty$. Terms proportional to $P_{-}^{2}$ in the normalization factors have been neglected, because they do not contribute to the highest power in $\nu$. The amplitudes $\mathrm{T}_{00}^{F}, \mathrm{~T}_{33}^{F}$ and $\mathrm{T}_{03}^{F}, \mathrm{~T}_{30}^{F}$ are a priori not of order $1 / \nu$, because $q_{\rho} \mathcal{K}^{a \rho}$ is of order $\nu$. But since the integrals

$$
\int_{\mathbb{R}^{3}} D^{3} z F\left(z_{+}, z_{-}, z_{k}\right)=0
$$

vanish, these amplitudes are also identical to zero.

In the above only the contractions of the helicity vectors with the Compton amplitude were considered. For the physical process, however, the corresponding projections for the leptonic tensors $L_{\mu \nu}^{1,2}$ have to be considered as well to see, which terms contribute to the physical $S$ matrix. Due to the fact that

$$
L_{\mu \nu}^{1} q_{1}^{\nu}=L_{\mu \nu}^{2} q_{2}^{\nu} \equiv 0
$$

holds all remaining terms in the projections $\mathrm{T}_{0 k}$ and $\mathrm{T}_{k 0}$ are annihilated.

In leading order in $\nu$ only the amplitudes $\mathrm{T}_{11}^{F}, \mathrm{~T}_{22}^{F}$ and $\mathrm{T}_{12}^{F 5}, \mathrm{~T}_{21}^{F 5}$ give a nonvanishing contribution in the extended Bjorken region, whereas other terms $\mathrm{T}_{k l}^{(5)}$ for $k, l=1,2,3$ are suppressed at least in $O(1 / \sqrt{\nu})$. The explicit calculation also shows that

$$
\mathrm{T}_{11}^{F}=\mathrm{T}_{22}^{F} \quad \text { and } \quad \mathrm{T}_{12}^{F 5}=-\mathrm{T}_{21}^{F 5}
$$

in leading order. Only two of the 16 amplitudes are relevant for $\nu \rightarrow \infty$. Similar results have been obtained in Ref. [38]. 
[1] F. J. Yndurain, Quantum Chromodynamics. An Introduction to the Theory of Quarks and Gluons (Springer, New York, 1983); Lectures on QCD. II: Applications, edited by F. Lenz, H. Grieshammer, and D. Stoll (Springer, Berlin, 1997).

[2] T. Muta, Foundations of QCD. An Introduction to Perturbative Methods in Gauge Theories (World Scientific, Singapore, 1987).

[3] D. Müller, D. Roboaschik, B. Geyer, J. Horejsi, and F. M. Dittes, Fortschr. Phys. 42, 101 (1994).

[4] X. Ji, Phys. Rev. Lett. 78, 610 (1997); Phys. Rev. D 55, 7114 (1997); J. Phys. G 24, 1181 (1998); A. V. Radyushkin, Phys. Lett. B 385, 333 (1996); Phys. Rev. D 56, 5524 (1997); I. I. Balitsky and A. V. Radyushkin, Phys. Lett. B 413, 114 (1997).

[5] J. Blümlein, B. Geyer, and D. Robaschik, Phys. Lett. B 406, 161 (1997).

[6] J. Blümlein, B. Geyer, and D. Robaschik, Nucl. Phys. B560, 283 (1999).

[7] J. Blümlein and D. Robaschik, Nucl. Phys. B581, 449 (2000).

[8] M. Vanderhaeghen, Eur. Phys. J. A 8, 455 (2000); K. Goeke, M. Polyakov, and M. Vanderhaeghen, Prog. Part. Nucl. Phys. 47, 401 (2001).

[9] ZEUS Collaboration, P. Saull, hep-ex/0003030; Abstract 564, EPS HEP Budapest, 2001.

[10] H1 Collaboration, C. Adloff et al., Phys. Lett. B 517, 47 (2001).

[11] HERMES Collaboration, A. Airapetian et al., Phys. Rev. Lett. 87, 182001 (2001).

[12] MAMI Collaboration, Nucl. Phys. A666, 44 (2000); MAMI Collaboration, J. Roche et al., Phys. Rev. Lett. 85, 708 (2000).

[13] G. Audit et al., TJNAF experiment 93-050; B. B. Wojtsekhovskii et al., TJNAF experiment 99-114; J. P. Chen et al., TJNAF experiment $00-110$.

[14] Similarly to the case of deep-inelastic nonforward scattering multiple hadron final states also emerge in deep-inelastic diffractive scattering [39] along the diffractive final state proton, which is assumed an isolated particle in the ideal case, Ref. [40].

[15] As has been shown recently in Ref. [40], also the case of deep inelastic diffractive scattering can be described following similar lines.

[16] S. A. Anikin and O. I. Zavialov, Ann. Phys. (N.Y.) 116, 135 (1978).

[17] B. Geyer, M. Lazar, and D. Robaschik, Nucl. Phys. B559, 339 (1999).

[18] B. Geyer, M. Lazar, and D. Robaschik, Nucl. Phys. B618, 99 (2001).

[19] D. J. Gross and S. B. Treiman, Phys. Rev. D 4, 1059 (1971).

[20] B. Geyer and M. Lazar, Nucl. Phys. B581, 341 (2000).

[21] R. I. Jaffe and X. Ji, Nucl. Phys. B375, 527 (1991).

[22] B. Geyer and M. Lazar, Phys. Rev. D 63, 094003 (2001).
[23] I. Balitsky and V. Braun, Nucl. Phys. B361, 93 (1991).

[24] For special choices of the nucleon spin vector, e.g., when directed along the momentum $P_{+}$, the second tensor structure may become identical to the former one up to subleading corrections of $O\left(M^{2} / \nu\right)$ as well known from the case of forward scattering, see, e.g., Ref. [27]. Although the second structure appears as kinematically suppressed it is still present and a WW-like relation between the twist- 2 contributions of the two amplitudes exists. For other choices of the spin vector this suppression does not occur.

[25] S. Wandzura and F. Wilczek, Phys. Lett. 72B, 195 (1977).

[26] Needless to say that the Lorentz decomposition of the complete Compton amplitude delivers higher twist corrections to the quantities $\mathrm{G}_{k}^{a}$ as well; in some cases twist-3 terms are present in the massless limit. These contributions are not dealt with in the present paper. We mention that in the case when quark and target mass corrections are taken into account all contributions may receive higher twist corrections starting with twist-3, as has been shown for the forward case in Ref. [27]. Integral relations between higher twist contributions do also exist.

[27] J. Blümlein and A. Tkabladze, Nucl. Phys. B553, 427 (1999); J. Phys. G 25, 1553 (1999); Nucl. Phys. B (Proc. Suppl.) 79, 541 (2000).

[28] J. Blümlein and D. Kochelev, Phys. Lett. B 381, 296 (1996); Nucl. Phys. B498, 285 (1997).

[29] P. Ball and M. Lazar, Phys. Lett. B 515, 131 (2001).

[30] M. Lazar, J. High Energy Phys. 05, 029 (2001).

[31] A. V. Belitsky and D. Müller, Nucl. Phys. B589, 611 (2000); N. Kivel, M. V. Polyakov, A. Schäfer, and O. V. Teryaev, Phys. Lett. B 497, 73 (2001); A. V. Radyushkin and C. Weiss, Phys. Rev. D 63, 114012 (2001); I. V. Anikin and O. V. Teryaev, Phys. Lett. B 509, 95 (2001).

[32] C. G. Callan and D. J. Gross, Phys. Rev. Lett. 22, 156 (1969).

[33] H. Georgi and H. D. Politzer, Phys. Rev. D 14, 1829 (1976).

[34] Th. Braunschweig, B. Geyer, and D. Robaschik, Ann. Phys. (Leipzig) 44, 407 (1987).

[35] I. Balitsky and V. Braun, Nucl. Phys. B311, 541 (1989).

[36] A. P. Prudnikov, Yu. A. Brychov, and O. I. Marichev, Integrals and Series. Vol. 2: Special Functions (Gordon and Breach Science Publishers, New York, 1992).

[37] H. Bateman and A. Erdelyi, Higher Transcendental Functions (McGraw-Hill, New York, 1953), Vol. 2.

[38] M. Diehl, T. Gousset, B. Pire, and J. P. Ralston, Phys. Lett. B 411, 193 (1997).

[39] ZEUS Collaboration, J. Breitweg et al., Eur. Phys. J. C 6, 43 (1999); H1 Collaboration, C. Adloff et al., Z. Phys. C 76, 613 (1997).

[40] J. Blümlein and D. Robaschik, Phys. Lett. B 517, 222 (2001). 